\title{
Time-evolution of bubble formation in a viscous liquid
}

\author{
Kou Yamada ${ }^{1}$, Hiroyuki Emori ${ }^{2}$, and Kiyoshi Nakazawa ${ }^{3}$ \\ ${ }^{1}$ Graduate School of Political Science, Waseda University, Nishiwaseda, Tokyo 169-8050, Japan \\ ${ }^{2}$ Department of Business Management, Shumei University, Chiba 276-0003, Japan \\ ${ }^{3}$ Department of Earth and Planetary Sciences, Tokyo Institute of Technology, Ookayama, Tokyo 152-8551, Japan
}

(Received September 11, 2007; Revised February 23, 2008; Accepted February 25, 2008; Online published July 4, 2008)

\begin{abstract}
Time-evolutions of bubble vesiculation in viscous liquid are characterized by two stages, i.e., the bubble formation stage and the bubble coalescence stage. We have focused on the former stage of bubble vesiculation and investigated numerically bubble formation in decompressed viscous liquid. Bubble formation consists of nucleation and growth of bubbles due to the exsolution of volatile elements from liquid. In order to describe the bubble formation processes, we have developed a theoretical model, taking into account the bubble size distribution and the viscosity of liquid, on the base of the model proposed by Toramaru (J. Geophys. Res., 100, 1913-1931, 1995). Numerical solutions show that the feature of bubble nucleation is significantly varied according to the magnitude of the liquid viscosity. In low viscosity cases, the growth of bubbles is mainly controlled by the diffusive flux of volatile molecules to bubbles. The number density of bubbles is sensitive to the diffusivity of volatile element in liquid. On the other hand, bubbles hardly grow in the case of the extremely high viscosity. Therefore, bubbles continue to be formed in order to reduce the volatile oversaturation of liquid. In the case of the intermediate viscosity, bubbles grow to the large size slowly by the viscous resistance. After the nucleation of bubbles ceases, the Ostwald ripening occurs and leads to the dissolution of small bubbles. The number density of bubbles varies greatly with time. The time-evolutions of the bubble size distribution have been also caluclated. In the case of the low and extreme high viscosity, the bubble size distribution evolves as an unimodal size distribution. In the case of the moderate viscosity, the size distribution of bubbles shows the distribution with a wider dispersion. These characteristic time-evolutions of the number density and size distribution of bubbles would be useful in evaluating material quantities such as the diffusivity, the viscosity, and the surface tension of liquid from experimental results. Furthermore our results provide the number density and size distribution of bubbles just before bubble coalescence occurs.
\end{abstract}

Key words: Bubble, nucleation, growth, viscous, Ostwald ripening, size distribution.

\section{Introduction}

The kinetics of phase change plays important roles in many fields of science and technology. Phase changes proceed through the nucleation and growth of new phase. Studies on the nucleation and growth are necessary to understand various physical phenomena for example, bubble formation in ascending magmas and condensation of solid dusts in cooling gas. Theoretical or experimental studies have been done so far to describe the phase transition kinetics (Lothe and Pound, 1962; Feder et al., 1966; Kikuchi, 1971; Anderson et al., 1980; Adams et al., 1984; Dillmann and Meier, 1991; Yamamoto et al., 2001) and one can now sketch out how phase change proceeds in nucleation processes.

In general, phase change proceeds through three successive stages. The first stage is the supersaturated state in which supersaturation rates of matters to make a new phase are increased by change in thermodynamic conditions such as cooling of temperature, decompression, or chemical reactions. The second stage is formation of new phase. Nuclei

Copyright (c) The Society of Geomagnetism and Earth, Planetary and Space Sciences (SGEPSS); The Seismological Society of Japan; The Volcanological Society of Japan; The Geodetic Society of Japan; The Japanese Society for Planetary Sciences; TERRAPUB. of new phase come out in the interior of parent phase. After this stage, as the third stage, the growth of nuclei proceeds. In the growth stage, interactions between nuclei are negligible due to smallness of nuclei. Then, a nucleus grows by the attachment of new phase molecules or expands by difference between its internal and external pressure. In latter stage, interactions between nuclei may be important. Nuclei grow to the large size via the coalescence between nuclei. Then, the relative velocity and the distance between nuclei which are related to the number density and size distribution of nuclei are important factors controlling the coalescence efficiency.

The nucleation and growth of nuclei have been theoretically investigated by many authors on the basis of the classical nucleation theory (Johnson and Mehl, 1939; Avrami, 1939, 1940, 1941; Yamamoto and Hasegawa, 1977; Toramaru, 1989, 1995; Pradell et al., 1998; Yamada et al., 2005). They constructed theoretical models to deduce total volume, final number density, or size distribution of population. As a result, in these studies, physical conditions or material quantities are estimated by the comparison with population of new phase observed in natural samples, for example, pyroclastic rocks (e.g., Toramaru, 2006) or meteorites (e.g., Tanaka et al., 2002). 
Bubble dynamics is one of important problems because the coexistence of gas bubbles with liquid remarkably changes properties of flow. Such two-phase flow is usually called multiphase flow. For example, magma ascending in conduit or rapid flow over a submerged body are observed as multiphase flow. The specific volume of matter is much larger in gas phase than in liquid phase. So, increase in the volume ratio of gas phase results in acceleration of the flow under gravity even if the mass ratio of gas is small (Papale, 2001). Besides, as another example, the drag coefficient in multiphase flow generally depends on the volume ratio of gas (Batchelor, 1967). In this paper, we will focus on bubble formation processes, that is, the bubble nucleation stage and the single growth stage. We will develop a theoretical model on the nucleation and growth of bubbles in a viscous liquid containing a volatile component to examine time-evolutions of the bubble size distribution and the bubble number density, which are used to speculate bubble vesiculation processes happened actually from natural samples (e.g., Proussevitch et al., 2007a, b). Our study will provide useful informations on initial conditions of the bubble coalescence stage.

In studies for condensation, Yamamoto and Hasegawa (1977) derived the equations describing the formation and growth of grains in cooling vapor composed of one species, taking into account the grain size distribution. They solved the equations analytically and obtained the number density of grains and the final grain size as a function of physical parameters such as the cooling rate and the surface energy of grain.

Quantitative description of the nucleation process in a viscous liquid was originally provided by Toramaru (1995). He constructed the bubble nucleation model in decompressing viscous incompressible magmas, taking into account the time-evolution of $\mathrm{H}_{2} \mathrm{O}$ concentration in magma. He numerically calculated the number density of bubbles and the supersaturation pressure at which bubble nucleation is virtually triggered. Further, he derived empirical expressions for the bubble number density and the duration time of nucleation by the usage of their numerical results. However, he assumed that all bubbles have equal size ignoring the bubble size distribution.

On the basis of Toramaru's model $(1989,1995)$, Yamada et al. (2005) developed the nucleation model in magmas with the low viscosity taking into account the size distribution of bubbles and derived approximate solutions to the bubble number density and the supersaturation pressure based on the analytical method of Yamamoto and Hasegawa (1977). Their results are as follows: The analytical solution provides number densities reproducing well the numerical values within a factor of 2 in wide ranges of parameters. The values of the bubble number density agree with those given by Toramaru (1995) within a factor of 10. In the case of the small viscosity, the size distribution of bubbles shows an unimodal distribution with the narrow dispersion. Since the assumption that all bubbles are equal in size is valid, the discrepancy between our results and those of Toramaru (1995) is observed within one order of magnitude.

As for bubble formation in magma, many laboratory experiments of vesiculation have been performed as well as theoretical studies (Hurwitz and Navon, 1994; Gardner et al., 1999, 2000; Gardner, 2007; Mangan and Sisson, 2000; Mangan et al., 2004a, b; Mourtada-Bonnefoi and Laporte, 1999, 2002, 2004). The experiments provided data on vesiculation parameters such as the time-evolution of the number density of bubbles, the bubble size distribution, and the supersaturation pressure. Yamada et al. (2005) indirectly estimated the surface tension at water-rhyolitic melt interface and the $\mathrm{H}_{2} \mathrm{O}$ diffusivity in rhyolitic melt from the comparison between their model and some experiments. The indirect estimates are consistent with the available measurements of the surface tension of the melt (Epel'baum et al., 1973; Khitarov et al., 1979; Bagdassarov et al., 2000) and the water diffusivity in the melt (Nowak and Behrens, 1997; Zhang, 1999; Zhang and Behrens, 2000).

In the model of Yamada et al. (2005), the effect of the viscosity on the bubble growth is assumed to be negligible. As pointed out by previous studies (Sparks, 1978; Proussevitch et al., 1993; Toramaru, 1995; Proussevitch and Sahagian, 1998), however, the growth of bubbles is controlled by the viscosity in high viscous melt and bubble nucleation is sensitive to the viscosity through the bubble growth. Hence, in this paper, we will investigate the non-equilibrium exsolution of gas in a decompressing viscous liquid. In order to describe the nucleation and growth of bubbles, we will develop a theoretical model, taking into account the size distribution of bubbles.

Our main aim of this paper is to examine how timeevolution of the bubble number density and size distribution of bubbles depend on material parameters including the surface tension, the volatile diffusivity, and the viscosity. This kind of study is fundamentally important for following reasons: (1) Quantitative relationships between observations (e.g., the bubble number density and the bubble size distribution) and unknown material parameters can be powerful tools to study material quantities and physical conditions from natural samples or experiments. (2) Once we obtain informations on time-evolutions of the bubble number density and bubble size distribution, we can easily incorporate them as initial conditions on the latter bubble vesiculation process, namely, bubble coalescence stage.

In Section 2, we will describe the basic equations, taking the bubble size distribution into account, where we basically follow the study of Toramaru (1995). In Section 3, we will show the simulations of the time-evolution of bubble formation and the behavior of the bubble number density. We will adopt parameter ranges similar to magmatic parameters to compare our results with those of Yamada et al. (2005) and Toramaru (1995). We will see the time-evolution of the bubble size distribution and check the validity of the assumption of equal bubble size in the model of Toramaru (1995). In Section 4, the conclusions of our study will be presented.

\section{Basic Equations Governing Nucleation and Growth of Bubbles}

\subsection{Nucleation and growth of bubbles}

We will describe basic equations governing the nucleation and growth of bubbles. In this paper, the temperature in fluid is assumed to be constant. Since the heat capacity 
of liquid is generally large, this assumption is valid for the case that the gas to liquid mass ratio is small. In this study we also assume that the surface tension, the viscosity, and the volatile diffusivity in liquid are constant.

We define the concentration of a volatile component expressed by $C$ as the number of volatile molecules per unit fluid volume. The activity of volatile species plays an important role in controlling the degree of supersaturation in fluid. The activity of mixture that contains a volatile material has been experimentally and theoretically examined by many studies in volcanology (Burnham, 1975; Ghiorso et al., 1983; Fogel and Rutherford, 1990; Papale, 1999). According to their results, the activity is proportional to the $m$-power of volatile concentration, namely $C^{m}$. The exponent $m$ depends on a volatile species. In the case that the volatile is $\mathrm{H}_{2} \mathrm{O}, m=2$ and in the case of $\mathrm{CO}_{2}$ for silicic magma, $m=1$.

Let us derive the equilibrium concentration $C_{\text {eq }}$ of a volatile component in liquid at the pressure $P$ from the chemical equilibrium condition. In an isothermal state, the equilibrium concentration is given, in terms of a concentration $C_{0}$ and the pressure $P_{0}$ at a reference saturation state, by (e.g., Toramaru, 1989, 1995)

$$
\frac{C_{\mathrm{eq}}}{C_{0}}=\left(\frac{P}{P_{0}}\right)^{1 / m} \exp \left[\frac{v_{1}}{m k_{\mathrm{B}} T}\left(P_{0}-P\right)\right],
$$

where $v_{1}$ is the volatile molecular volume in liquid, $T$ is the temperature, and $k_{\mathrm{B}}$ is the Boltzmann's constant. In deriving above equation, the volatile molecular volume $v_{\mathrm{l}}$ is set to be constant and the gas phase is assumed to be ideal.

We introduce the saturated concentration of a volatile component $C_{\text {in }}$ equilibrated with a spherical bubble. The internal pressure in a bubble is different from the pressure in surrounding liquid due to the surface tension in the case where a bubble and liquid contact through a curved interface. Since liquid with the concentration $C_{\text {in }}$ and the pressure $P$ keeps the chemical equilibrium state with a bubble in which the internal pressure is $P_{\text {gas }}$, the saturated concentration is given by

$$
\frac{C_{\mathrm{in}}}{C_{0}}=\left(\frac{P_{\mathrm{gas}}}{P_{0}}\right)^{1 / m} \exp \left[\frac{v_{\mathrm{l}}}{m k_{\mathrm{B}} T}\left(P_{0}-P\right)\right] .
$$

We set a reference concentration $C_{0}$ to be the initial value of volatile concentration in fluid and assume that the fluid is saturated at $t=0$ (i.e., $P=P_{0}$ ). Under the perfect thermal equilibrium condition, bubbling of a volatile component begins just at the equilibrium pressure $P_{0}$. In real systems, however, the surface energy at an interface between liquid and gas suppresses the nucleation of bubbles and the fluid remains in the super-saturated state for a long period of time. Then, we introduce the supersaturation ratio defined by

$$
S=\frac{C}{C_{\mathrm{eq}}}
$$

The equilibrium concentration decreases with decompression. It is noted that $S$ is greater than unity in supersaturated liquid.

In the super-saturated state, nucleation of new phase proceeds through the formation of nuclei whose radius is larger than that of a critical nuclei. This nucleation of bubbles in a metastable phase is controlled by thermodynamic fluctuations. According to the classical nucleation theory (Feder et al., 1966; Lifshitz and Pitaevskii, 1981), the nucleation rate is given by

$$
J=\frac{D C^{2}}{4 \pi m} \sqrt{\frac{k_{\mathrm{B}} T}{\gamma}}\left(1-S^{-m}\right) \exp \left[-\frac{4 \pi \gamma}{3 k_{\mathrm{B}} T} R_{\mathrm{cr}}^{2}\right],
$$

where $D$ is the diffusion coefficient of a volatile component in liquid, $\gamma$ is the surface tension, and $R_{\mathrm{cr}}$ is the radius of a critical nucleus given by

$$
R_{\mathrm{cr}}=\frac{2 \gamma}{P\left(S^{m}-1\right)} \text {. }
$$

Decompression leads to the increase in the supersaturation ratio and the formation of bubbles. Then, in homogeneous liquid, bubbles are uniformly distributed, namely the distance from a bubble to neighboring bubbles is approximately equal. At the former stage, interactions between bubbles are negligible because the radius of a bubble is much smaller than the mean distance between bubbles. However, at the later stage, when bubbles grow enough, bubble coalescence would occur and as a consequence the size distribution of bubbles changes. We principally consider the nucleation and growth stage of bubbles before bubble coalescence occurs.

The growth of a nucleated bubble is controlled by the diffusion of volatile molecules in super-saturated liquid. We derive an equation describing the time variation of the number of volatile molecules in a single bubble, $n$. Since the number of volatile molecules in a bubble increases by the diffusive flux toward its surface in super-saturated liquid, its time-evolution is given by

$$
\frac{\mathrm{d} n\left(t, t^{\prime}\right)}{\mathrm{d} t}=4 \pi R^{2} I
$$

where $R$ is the bubble radius and $I$ is the diffusive flux toward the bubble. Note that $R$ and $n$ are dependent on the time $t^{\prime}$ indicating when the bubble was nucleated as well as present time $t$. Assuming a steady state, the spherically symmetric distribution round a bubble of radius $R$ is given by the solution of the diffusion equation with boundary conditions that the concentrations are equal to $C$ and $C_{\text {in }}$ at infinity and the bubble surface, respectively. Using the solution, the diffusion flux is given as $I=D\left(C-C_{\text {in }}\right) / R$. Proussevitch et al. (1993) indicated that gradient of volatile concentrations at a bubble-liquid interface changes from steep at the early time to flat at the late time. Actually, the volatile concentration in liquid varies with time and the spatial gradient of volatile concentration surrounding a bubble does not become linear but hyperbolic (Proussevitch et al., 1993). In the diffusive flux derived here, the bubble radius $R$ is used as the characteristic diffusion length and $C-C_{\text {in }}$ is used as characteristic difference in the volatile concentration. Thus, since the radius of the bubble is small around its nucleation time $t^{\prime}$, the diffusion flux is large. On the other hand, when bubbles grow up to the large size and the volatile concentration in liquid decreases with time, the diffusive flux toward a bubble diminishes. Therefore, this expression of the diffusive flux approximately reproduces the 
time evolving diffusive flux on the bubble-liquid interface (Yamada, 2005). Substituting the stationary solution of the diffusive flux into Eq. (6), we obtain

$$
\frac{\mathrm{d} n\left(t, t^{\prime}\right)}{\mathrm{d} t}=4 \pi R D\left(C-C_{\text {in }}\right) .
$$

In this study, we simply apply the mean-field approximation to evaluate the spatial volatile concentration within liquid (e.g., Pradell et al., 1998). Under the mean-field approximation, the volatile concentration in liquid surrounding bubbles is deduced from the conservation of the total number of volatile molecules as (Yamamoto and Hasegawa, 1977)

$$
C(t)=C_{0}-\int_{0}^{t} n\left(t, t^{\prime}\right) J\left(t^{\prime}\right) \mathrm{d} t^{\prime} .
$$

Decompression causes the pressure difference between liquid and gas which leads to the expansion of gas bubbles. The pressure difference balances with the viscosity, the surface tension, and the inertial force (Proussevitch et al., 1993; Toramaru, 1995; Lyakhovsky et al., 1996; Proussevitch and Sahagian, 1998; Lensky et al., 2001):

$$
P_{\mathrm{gas}}=P+\frac{2 \gamma}{R}+\frac{4 \eta}{R} \dot{R}+\rho_{\mathrm{l}}\left(2 \dot{R}^{2}+R \ddot{R}\right)
$$

In Eq. (9), $\rho_{1}$ is the liquid density. The above equation was derived by many investigators with the assumption of the liquid incompressibility. Furthermore, the applicability of Eq. (9) to the bubble growth in compressible liquid was rigorously verified by Yamada et al. (2006). In the case that the expansion rate of bubbles, $\dot{R}$, is considerably smaller than the sound velocity $c_{\mathrm{s}}$, the last two terms on the right hand side of Eq. (9) can be omitted. These terms, which we call the inertial term henceforth, have an order of $\rho_{1} \dot{R}^{2}$ while the pressure term is about $\rho_{1} c_{\mathrm{s}}^{2}$. For rough estimation, putting appropriate values $\left(\dot{R} \simeq 1 \times 10^{-4} \mathrm{~cm} / \mathrm{s}\right.$ and $c_{\mathrm{s}} \simeq$ $1 \times 10^{5} \mathrm{~cm} / \mathrm{s}$ ) into the inertial term, the ratio of the inertial term to the pressure term, which corresponds to the Mach number, is much smaller than unity. Thus, neglecting the inertial term, the time variation of the bubble expansion is given by (Proussevitch et al., 1993; Toramaru, 1995; Proussevitch and Sahagian, 1998)

$$
\frac{\mathrm{d} R}{\mathrm{~d} t}=\frac{R}{4 \eta}\left(P_{\mathrm{gas}}-P-\frac{2 \gamma}{R}\right) .
$$

The internal pressure in a bubble is related to $R$ and $n$ through the equation of state of ideal gas:

$$
\frac{4 \pi}{3} R^{3} P_{\mathrm{gas}}=n k_{\mathrm{B}} T
$$

The growth of bubbles is described by Eqs. (7) and (10). Assuming that a critical nucleus is in equilibrium chemically and mechanically with surrounding liquid, the internal pressure in the critical nucleus is brought from Eq. (10) as

$$
P_{\mathrm{gas}}=P+\frac{2 \gamma}{R_{\mathrm{cr}}}
$$

We integrate Eqs. (7) and (10) with the initial conditions, $R\left(t^{\prime}, t^{\prime}\right)=R_{\mathrm{cr}}$, and Eq. (12).

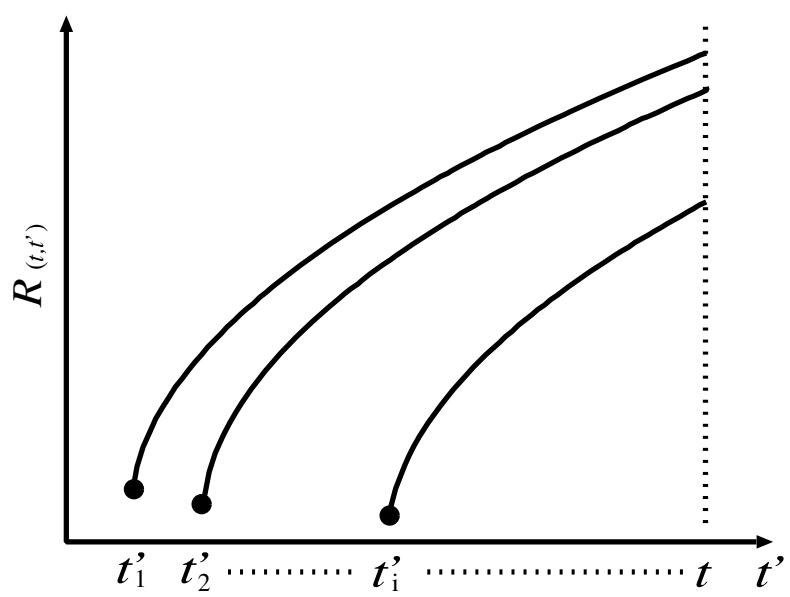

Fig. 1. Schematic time-evolution of the radius of bubbles which are nucleated at $t^{\prime}=t_{i}^{\prime}$.

Bubbles nucleated earlier grow larger than those nucleated later. Figure 1 shows schematic time-evolutions of radii of bubbles nucleated at different time $t^{\prime}$. The size distribution of bubbles arises from the varieties of the nucleation time among bubbles. As seen in Eq. (8), accurate information on the bubble size distribution is required to calculate the time-evolution of volatile concentration. In the numerical calculation by Toramaru (1995), he used a further simplified equation, in which bubbles have only one monotonic size. On the other hand, we directly solve Eq. (8), taking into account the size distribution of bubbles. The bubble nucleation process is described by Eqs. (1)-(5), (7), (8), and (10). In our simulations, bubbles with various sizes can be observed at time $t$. Then, in some cases, bubbles become smaller than the critical radius. We regard these bubbles as bubbles dissoluting to liquid and the number density of smaller bubbles than the critical radius is set to be zero.

The decline in the supersaturation ratio strongly reduces the nucleation rate and as a result bubble nucleation ceases. The number density of bubbles at time $t$ is given by

$$
N(t)=\int_{0}^{t} J\left(t^{\prime}\right) \mathrm{d} t^{\prime}
$$

In addition we introduce the cumulative number density of bubbles defined by

$$
N(>R)=\int_{R}^{\infty} F(R) \mathrm{d} R,
$$

where $F(R)$ is the size distribution of bubbles defined by

$$
F\left(R\left(t, t^{\prime}\right), t\right)=J\left(t^{\prime}\right)\left|\frac{\mathrm{d} R\left(t, t^{\prime}\right)}{\mathrm{d} t^{\prime}}\right|^{-1} .
$$

Equation (15) comes from the conservation of the number of bubbles between at the nucleation time $t^{\prime}$ and any time $t$ at which bubbles grow up to radius $R\left(t, t^{\prime}\right)$. The cumulative number density $N(>R)$ represents the total number density of bubbles with a larger radius than $R$. 


\subsection{Non-dimensional forms}

For the later convenience, we transform the basic equations mentioned in previous subsection into nondimensional forms. In this study, we consider the case where decompression proceeds at a constant rate. Then, the pressure of liquid with a constant decompression rate is given by $P=P_{0}-\dot{P} t$. We introduce non-dimensional time $x$ defined by

$$
x=\frac{t}{t_{P}},
$$

where $t_{P}$ is the decompression time defined by $P_{0} / \dot{P}$.

Now, we introduce the non-dimensional volatile concentration $\tilde{C}$, the pressure $\tilde{P}$, and the bubble radius $\tilde{R}$, defined by

$$
\begin{aligned}
& \tilde{C}(x)=\frac{C(t)}{C_{0}}, \\
& \tilde{P}(x)=\frac{P(t)}{P_{0}},
\end{aligned}
$$

and

$$
\tilde{R}\left(x, x^{\prime}\right)=\frac{R\left(t, t^{\prime}\right)}{r_{1}},
$$

respectively, where $r_{1}$ is the mean distance between neighboring volatile molecules in a bubble defined by

$$
\frac{4 \pi}{3} r_{1}^{3}=\frac{k_{\mathrm{B}} T}{P_{0}} .
$$

From Eqs. (16)-(19), the basic Eqs. (7), (8), and (10) are rewritten as

$$
\begin{aligned}
& \frac{\mathrm{d} n\left(x, x^{\prime}\right)}{\mathrm{d} x}=3 \alpha \Lambda \tilde{R}\left(\tilde{C}-\tilde{C}_{\text {in }}\right), \\
& \frac{\mathrm{d} \tilde{R}\left(x, x^{\prime}\right)}{\mathrm{d} x}=\frac{\Theta}{4} \tilde{R}\left(\tilde{P}_{\mathrm{gas}}-\tilde{P}-\frac{2 \tilde{\gamma}}{3 \tilde{R}}\right),
\end{aligned}
$$

and

$$
\tilde{C}(x)=1-\int_{0}^{x} n\left(x, x^{\prime}\right) \tilde{J}\left(x^{\prime}\right) \mathrm{d} x^{\prime},
$$

respectively. Note that $\tilde{C}_{\text {in }}$ and $\tilde{P}_{\text {gas }}$ denote the nondimensional forms of the interfacial concentration and the internal pressure in a bubble, respectively. The parameters $\alpha, \Lambda, \tilde{\gamma}$, and $\Theta$ in the above equations are defined as

$$
\begin{aligned}
& \alpha=\frac{4 \pi r_{1}^{3} C_{0}}{3}, \\
& \Lambda=\frac{D t_{P}}{r_{1}^{2}}, \\
& \tilde{\gamma}=\frac{4 \pi r_{1}^{2} \gamma}{k_{\mathrm{B}} T},
\end{aligned}
$$

and

$$
\Theta=\frac{P_{0} t_{P}}{\eta},
$$

respectively. The parameters $\alpha, \Lambda, \tilde{\gamma}$, and $\Theta$ represent the ratio of the volume occupied by a single volatile molecule in the gas phase to that in the liquid phase, the ratio of the decompression time to the diffusion time for the distance $r_{1}$, the ratio of the surface energy of a hypothetical surface belonging to one volatile molecule to the thermal energy, and the ratio of the decompression time to the viscous relaxation time given by $\eta / P_{0}$, respectively. Furthermore, $\tilde{J}(x)$ is the non-dimensional nucleation rate written by

$$
\tilde{J}(x)=\frac{3 \alpha \Lambda}{8 m \sqrt{\pi^{3} \tilde{\gamma}}} \tilde{C}^{2}\left(1-S^{-m}\right) \exp \left(-\frac{\tilde{\gamma}}{3} \tilde{R}_{\mathrm{cr}}^{2}\right),
$$

where $\tilde{R}_{\text {cr }}$ is the non-dimensional radius of the critical nucleus. Then, the supersaturation ratio is also expressed as

$$
S(x)=\tilde{C}(1-x)^{-1 / m} \exp \left(-\frac{\beta x}{m}\right),
$$

where $\beta$ is the ratio of the volume occupied by a single volatile molecule in the gas phase to its molecular volume in the liquid phase defined by

$$
\beta=\frac{3 v_{1}}{4 \pi r_{1}^{3}}
$$

In addition, noted that the dimensionless number density of bubbles is

$$
\frac{N(x)}{C_{0}}=\int_{0}^{x} \tilde{J}\left(x^{\prime}\right) \mathrm{d} x^{\prime} .
$$

The basic equations include two differential equations and one integral equation. As for our numerical simulation, Eqs. (21) and (22) are integrated by the secondorder Runge-Kutta method with the initial conditions, $\tilde{R}\left(x^{\prime}, x^{\prime}\right)=\tilde{R}_{\mathrm{cr}}\left(x^{\prime}\right)$ and $\tilde{P}_{\text {gas }}=\tilde{P}+2 \tilde{\gamma} / 3 \tilde{R}_{\mathrm{cr}}$. The integration of Eq. (23) is calculated according to the trapezoidal rule. The governing equations contain six parameters $\alpha, \beta$, $\Lambda, \Theta, \tilde{\gamma}$, and $m$. The main notations used in this paper are listed in Table 1.

The bubble radius and a number of volatile molecules within bubbles are functions of $t$ and $t^{\prime}$. To obtain accurately the size distribution of bubbles, we have to calculate the growth of bubbles nucleated at different $t^{\prime}$ as shown in Fig. 1. In calculations, the time $t^{\prime}$ is discretely given such as $t^{\prime}=\left\{t_{1}^{\prime}, t_{2}^{\prime}, \cdots, t_{i}^{\prime}, \cdots, t\right\}$, where $t_{1}^{\prime}<t_{2}^{\prime}<\cdots<t_{i}^{\prime}<$ $\cdots<t$. Then, a time step between $t_{i-1}^{\prime}$ and $t_{i}^{\prime}$ is chosen small enough to carry out a stable calculation. In this study, we set it to be less than $1 / 3 \alpha \Lambda$ and $4 / \Theta$. The dimensionless parameters $1 / 3 \alpha \Lambda$ and $4 / \Theta$ correspond to the typical diffusive growth time and expansion time of bubbles in viscous liquid, respectively. The time step is usually given by $1 / 3 \alpha \Lambda$. As known later, $\Lambda$ is an order of $10^{6-8}$. Then, the time step is extremely small. If we follow time-evolutions of bubbles nucleated at each time, $t_{i}^{\prime}$, in the straightforward way, we must treat the huge number of bubbles at the late time. This needs vast memories and CPU time of a single computer. So, on the way of simulation, we group data of some bubbles nucleated at the earlier stage and thin down the number of bubbles whose growth we should calculate. By means of this way, memory and CPU time can be saved.

Before observing numerical results of bubble nucleation, we consider the likelihood of bubble coalescence. Connecting bubbles have been reported from observations of pyroclastic rocks (e.g., Klug and Cashman, 1994; Noguchi et al., 2006; Shimano and Nakada, 2006) and by experiments (Larsen and Gardner, 2000; Larsen et al., 2004). Therefore, it is expected that bubbles coalesce each other during 
Table 1. Main notation list.

\begin{tabular}{|c|c|}
\hline Parameter & Definition \\
\hline$a$ & Dimensionless parameter defined by Eq. (37). \\
\hline$b$ & Dimensionless parameter defined by Eq. (40). \\
\hline$C$ & Volatile concentration in liquid, number $/ \mathrm{m}^{3}$. \\
\hline$\tilde{C}$ & Dimensionless form of $C, C / C_{0}$ \\
\hline$C_{\text {eq }}$ & $\begin{array}{l}\text { Equilibrium concentration at the pressure } P \text {, } \\
\text { number } / \mathrm{m}^{3} \text {. }\end{array}$ \\
\hline$C_{\text {in }}$ & $\begin{array}{l}\text { Equilibrium concentration at liquid-spherical } \\
\text { bubble interface, number } / \mathrm{m}^{3} \text {. }\end{array}$ \\
\hline$\tilde{C}_{\text {in }}$ & Dimensionless form of $C_{\mathrm{in}}, C_{\mathrm{in}} / C_{0}$ \\
\hline$C_{0}$ & Initial concentration in liquid, number $/ \mathrm{m}^{3}$. \\
\hline$D$ & Volatile diffusivity in liquid, $\mathrm{m}^{2} / \mathrm{s}$. \\
\hline$J$ & Nucleation rate, number $/ \mathrm{m}^{3} / \mathrm{s}$. \\
\hline$\tilde{J}$ & Dimensionless form of $J, J t_{P} / C_{0}$. \\
\hline$\tilde{J}_{0}$ & $\begin{array}{l}\text { Dimensionless form of the zero-th order } \\
\text { nucleation rate given by Eq. (36). }\end{array}$ \\
\hline$m$ & Exponent of the activity. \\
\hline$N$ & Number density of bubbles, number $/ \mathrm{m}^{3}$. \\
\hline$N(>R)$ & $\begin{array}{l}\text { Cumulative number density of bubbles } \\
\text { whose size is larger than } R \text {, number } / \mathrm{m}^{3} \text {. }\end{array}$ \\
\hline$n$ & $\begin{array}{l}\text { Number of volatile molecules in a bubble, } \\
\text { number. }\end{array}$ \\
\hline$P$ & Pressure, Pa. \\
\hline$\tilde{P}$ & Dimensionless form of $P, P / P_{0}$. \\
\hline$P_{\text {gas }}$ & Internal pressure in a bubble, $\mathrm{Pa}$. \\
\hline$\tilde{P}_{\text {gas }}$ & Dimensionless form of $P_{\text {gas }}, P_{\text {gas }} / P_{0}$. \\
\hline$P_{0}$ & Initial pressure, $\mathrm{Pa}$ \\
\hline$\dot{P}$ & Decompression rate, $\mathrm{Pa} / \mathrm{s}$. \\
\hline$R$ & Bubble radius, $\mathrm{m}$. \\
\hline$\tilde{R}$ & Dimensionless form of $R, R / r_{1}$. \\
\hline$R_{\mathrm{cr}}$ & Radius of the critical bubbles, $\mathrm{m}$. \\
\hline$\tilde{R}_{\mathrm{cr}}$ & Dimensionless form of $R_{\mathrm{cr}}, R_{\mathrm{cr}} / r_{1}$ \\
\hline$r_{1}$ & Scaling constant defined by Eq. (20). \\
\hline$S$ & Supersaturation ratio, $C / C_{\mathrm{eq}}$ \\
\hline$T$ & Temperature, $\mathrm{K}$. \\
\hline$t$ & Actual time, s. \\
\hline$t^{\prime}$ & Time at which a bubble is nucleated, s. \\
\hline$t_{P}$ & Decompression time $P_{0} / \dot{P}$, s. \\
\hline$v_{1}$ & $\begin{array}{l}\text { Volume of a single volatile molecule in liquid, } \\
\mathrm{m}^{3} \text {. }\end{array}$ \\
\hline$x$ & Dimensionless time of $t, t / t_{P}$. \\
\hline$\alpha$ & Dimensionless parameter defined by Eq. (24). \\
\hline$\beta$ & Dimensionless parameter defined by Eq. (30). \\
\hline & $\begin{array}{l}\text { Relation between } \beta \text { and the parameter } \alpha_{2} \\
\text { used in Toramaru (1995): } \alpha_{2}=\beta\end{array}$ \\
\hline$\gamma$ & Surface tension of liquid, N/m. \\
\hline$\tilde{\gamma}$ & $\begin{array}{l}\text { Dimensionless parameter defined by Eq. (26). } \\
\alpha_{1} \text { (Toramaru, 1995) }=4 \tilde{\gamma}^{3} / 27 \text {. }\end{array}$ \\
\hline$\eta$ & Viscosity of liquid, Pa.s. \\
\hline$\Theta$ & $\begin{array}{l}\text { Dimensionless parameter defined by Eq. (27). } \\
\alpha_{4} \text { (Toramaru, 1995) }=\Theta / 4 \text {. }\end{array}$ \\
\hline$\Lambda$ & $\begin{array}{l}\text { Dimensionless parameter defined by Eq. (25). } \\
\alpha_{3} \text { (Toramaru, 1995) }=9 \alpha \Lambda / 4 \tilde{\gamma}^{2} \text {. }\end{array}$ \\
\hline
\end{tabular}
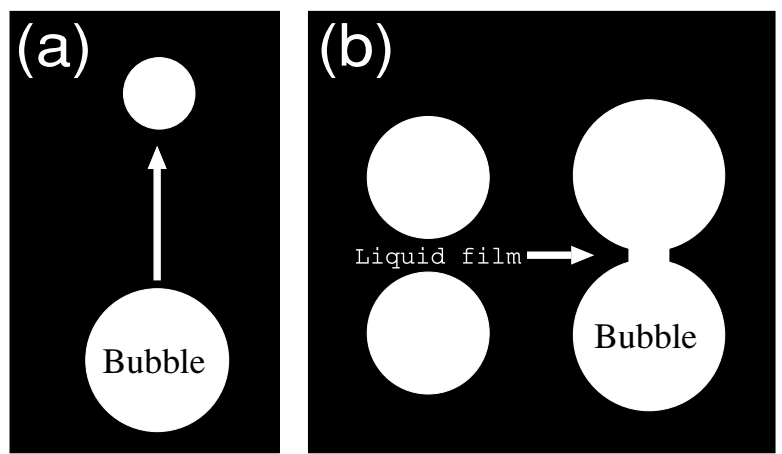

Fig. 2. Schematic bubble coalescence. In Panel (a), the collision of binary buoyant bubbles with the relative velocity is shown. Panel (b) shows the coalescence of expanding bubbles. As bubbles expand owing to decompression and approach each other, liquid film between them is thinning and two bubbles coalesce.

the bubble growth process. Then, there are two types of bubble coalescence namely, the collision of buoyant bubbles with the relative velocity (see Fig. 2(a)) or the coalescence as a result of the expansion of neighboring bubbles (see Fig. 2(b)).

Let us consider the first case. The drag is exerted on buoyant bubbles as they move through viscous liquid. Using Stokes' formula (Landau and Lifshitz, 1987), the velocity of moving bubbles $u_{\mathrm{b}}$ in liquid is given by

$$
u_{\mathrm{b}}=6 \times 10^{-8} \mu \mathrm{m} / \mathrm{s}\left(\frac{R}{1 \mu \mathrm{m}}\right)^{2}\left(\frac{\eta}{1 \times 10^{5} \mathrm{~Pa} \cdot \mathrm{s}}\right)^{-1}
$$

where we used the value of silicic magma for the viscosity and the liquid density is assumed to be much larger than the gas density within bubbles. As seen later, the typical size of nucleated bubbles ranges from 0.1 to $1 \mu \mathrm{m}$. Hence even if the relative velocity and the mean distance between two bubbles are almost equal to $u_{\mathrm{b}}$ and $R$, respectively, the collision time is much longer than the decompression time which is on the order of a few hundreds second. This implies that collision between small bubbles would be negligible.

When two expanding bubbles approach each other, liquid film between them is thinning and eventually cut off. Since the behavior of such coalescence is strongly dependent upon the motion of liquid around bubbles, this problem is considerably complex and it is beyond this study. Blower (2001) indicated that permeability is zero below the void ratio 0.3 , using a numerical model for mono-disperse bubbles placed at random positions. Moreover, the experimental measurement of permeability for samples produced by decompression experiment also showed that permeability is very low for samples with the porosity less than 45 vol.\% (Takeuchi et al., 2005). In this study, we assume that bubbles never unite when the void ratio is less than 0.3 . The numerical simulations are performed until the void ratio is equal to 0.3 or non-dimensional time $x$ is equal to unity. 


\section{Numerical Results}

\subsection{Behavior of bubble formation}

To observe the behavior of bubble formation, we numerically solve the basic equations in the case with following parameters:

$$
\left\{\begin{aligned}
m=2 & \\
\alpha= & 5 \times 10^{-1}\left(\frac{r_{1}}{3.4 \times 10^{-10} \mathrm{~m}}\right)^{3}\left(\frac{C_{0}}{3 \times 10^{27} \mathrm{~m}^{-3}}\right) \\
\beta= & 1.2 \times 10^{-1}\left(\frac{v_{1}}{2 \times 10^{-29} \mathrm{~m}^{3}}\right)\left(\frac{r_{1}}{3.4 \times 10^{-10} \mathrm{~m}}\right)^{-3} \\
\tilde{\gamma}= & 8.8 \times 10^{-1}\left(\frac{r_{1}}{3.4 \times 10^{-10} \mathrm{~m}}\right)^{2}\left(\frac{\gamma}{0.01 \mathrm{~N} / \mathrm{m}}\right) \\
& \quad\left(\frac{T}{1200 \mathrm{~K}}\right)^{-1}=9 \times 10^{7}\left(\frac{D}{1 \times 10^{-13} \mathrm{~m}^{2} / \mathrm{s}}\right)\left(\frac{t_{P}}{100 \mathrm{~s}}\right) \\
& \cdot\left(\frac{r_{1}}{3.4 \times 10^{-10} \mathrm{~m}}\right)^{-2} \\
\Theta= & 1 \times 10^{7}\left(\frac{P_{0}}{100 \mathrm{MPa}}\right)\left(\frac{t_{P}}{100 \mathrm{~s}}\right)\left(\frac{\eta}{1 \times 10^{3} \mathrm{~Pa} \cdot \mathrm{s}}\right)^{-1}
\end{aligned}\right.
$$

We call above values as reference values. In Fig. 3, timeevolutions of the volatile concentration (a), the nucleation rate (b), and the bubble number density (c) are illustrated.

Initially (at $x=0$ ), the equilibrium concentration is equal to the initial concentration $C_{0}$ and monotonously decreases with time, whereas the volatile concentration maintains $C_{0}$ until bubble nucleation occurs. After bubble nucleation ceases, the volatile concentration significantly decreases owing to the bubble growth and tends to an equilibrium value. In Fig. 3(b), the nucleation rate attains a maximum when the concentration decreases slightly (at $x=7.4 \times 10^{-2}$ ). The ratio of the duration time of nucleation (the half-value width of $J(t)$ ) to the decompression time is approximately $4 \times 10^{-3}$. This small ratio means that the nucleation proceeds at an instant. We can see from Fig. 3(c) that the non-dimensional number density of bubbles increases until $x=7.4 \times 10^{-2}$ and attains a maximum value, $3.1 \times 10^{-9}$ at $x=7.4 \times 10^{-2}$. After that, it slightly decreases. The supersaturation ratio decreases after $x=7.4 \times 10^{-2}$ through the decrease in the volatile concentration. Then, the critical radius increases (see Eq. (5)) and some bubbles become smaller than the critical radius again. Such bubbles are unstable and dissolve in liquid. As a result, the number density of bubbles decreases. This phenomenon is the Ostwald ripening (Lifshitz and Pitaevskii, 1981).

Let us see the behavior of bubble formation in the case of the medium viscosity. In Fig. 4, the parameter $\Theta$ is $2 \times 10^{4}$. Note that the values of other parameters are same as reference values. Time-evolutions of the volatile concentration (a), the nucleation rate (b), and the number density of bubbles (c) are shown in Fig. 4. It is found from Fig. 4(b) that the nucleation rate has a sharp maximum at $x=8.5 \times 10^{-2}$. The time when the nucleation rate attains a maximum is later than that in Fig. 3, in this case. A reason for this delay is that the growth of bubbles is suppressed by the viscosity. Then, the number density of nu-

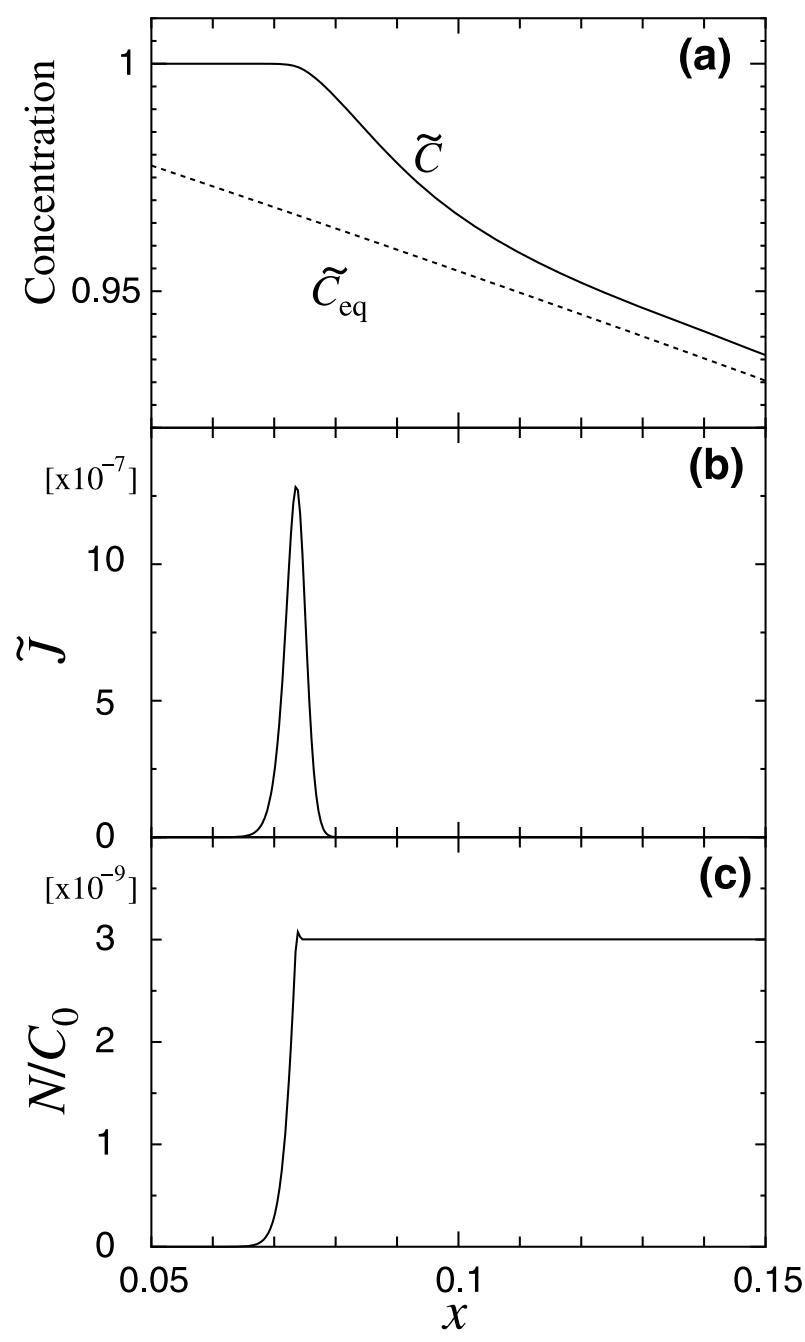

Fig. 3. Time-evolutions of the volatile concentration (a), the nucleation rate (b), and the number density of bubbles (c) in the case of reference values $\left(m=2, \alpha=0.5, \beta=1.2 \times 10^{-1}, \tilde{\gamma}=8.8 \times 10^{-1}, \Lambda=9 \times 10^{7}\right.$, and $\Theta=1 \times 10^{7}$ ). This corresponds to the low viscosity system. In Panel (a), the solid and dotted lines indicate the volatile concentration $C$ and the equilibrium value $C_{\text {eq }}$, respectively.

cleated bubbles is larger than that in Fig. 3. Consequently, the supersaturated liquid rapidly tends to the equilibrium state after the nucleation (see in Panel (a)). In Fig. 4(c), the number density of bubbles considerably decreases after it attains maximum. In highly viscous liquid, smaller bubbles cannot grow rapidly and they disappear due to the Ostwald ripening. Toramaru's model, in which all bubbles are assumed to have the same size, cannot observe the decrease in the bubble number density due to the Ostwald ripening. The disappearance of some small bubbles can be described because our study takes the size distribution of bubbles into account.

In the case of the higher viscosity, for $\Theta=1 \times 10^{2}$, timeevolutions of the volatile concentration (a), the nucleation rate (b), and the number density of bubble (c) are shown in Fig. 5. As found from Fig. 5(a) and (b), the volatile concentration decreases very slow but does not approach to the equilibrium concentration. The nucleation rate rapidly increases at the time as same as that in former two cases. 


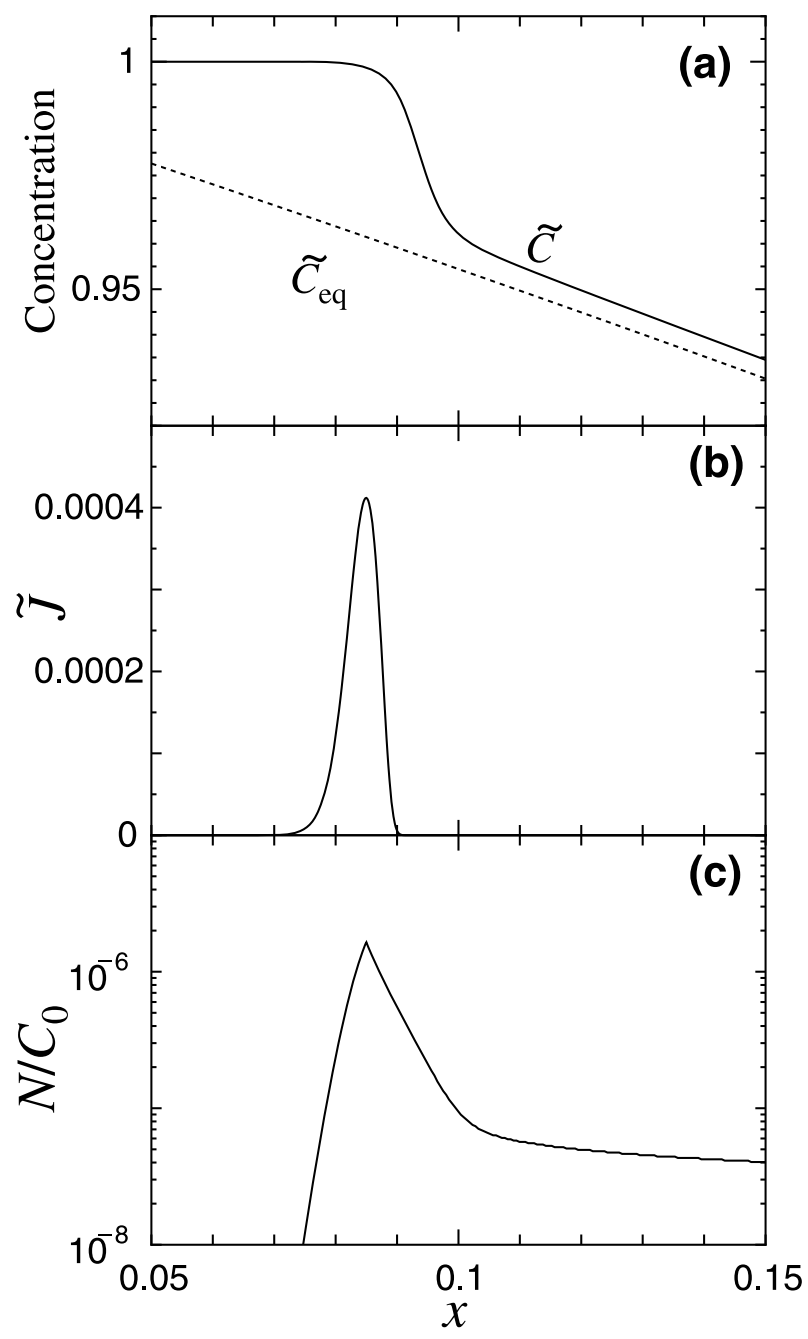

Fig. 4. Time-evolutions of the volatile concentration (a), the nucleation rate (b), and the number density of bubbles (c) for $\Theta=2 \times 10^{4}$. This corresponds to the medium viscosity system. Other parameters are equal to reference values. In Panel (a), the solid and dotted lines indicate the volatile concentration $C$ and the equilibrium value $C_{\text {eq }}$, respectively. The volatile concentration decreases after the nucleation of bubbles (at $x=8.5 \times 10^{-2}$ ) and rapidly approaches to the equilibrium concentration. The bubble number density considerably decreases owing to the Ostwald ripening after the nucleation.

However, it should be noted that the nucleation rate becomes larger than former two cases. Another difference is that bubble nucleation never ceases even if the volatile concentration decreases. The high viscosity hinders the bubble growth. As a consequence, volatile molecules in liquid are consumed only by the nucleation of bubbles. It is concluded that bubble nucleation in liquid with the high viscosity evolves as keeping the large supersaturation ratio to some extent.

Time-evolutions of the nucleation rate in the case of $\Theta=1 \times 10^{4}(\mathrm{a}), 2.5 \times 10^{3}(\mathrm{~b})$, and $1 \times 10^{3}(\mathrm{c})$ are shown in Fig. 6. As seen in Fig. 6, the duration time of nucleation is prolonged with decreasing $\Theta$. The higher viscosity prevents bubbles from growing. The prohibition of the bubble growth leads to the slight decrease in the volatile concentration. Therefore, the supersaturated state is predominately released by the nucleation of bubbles. When bubbles grow

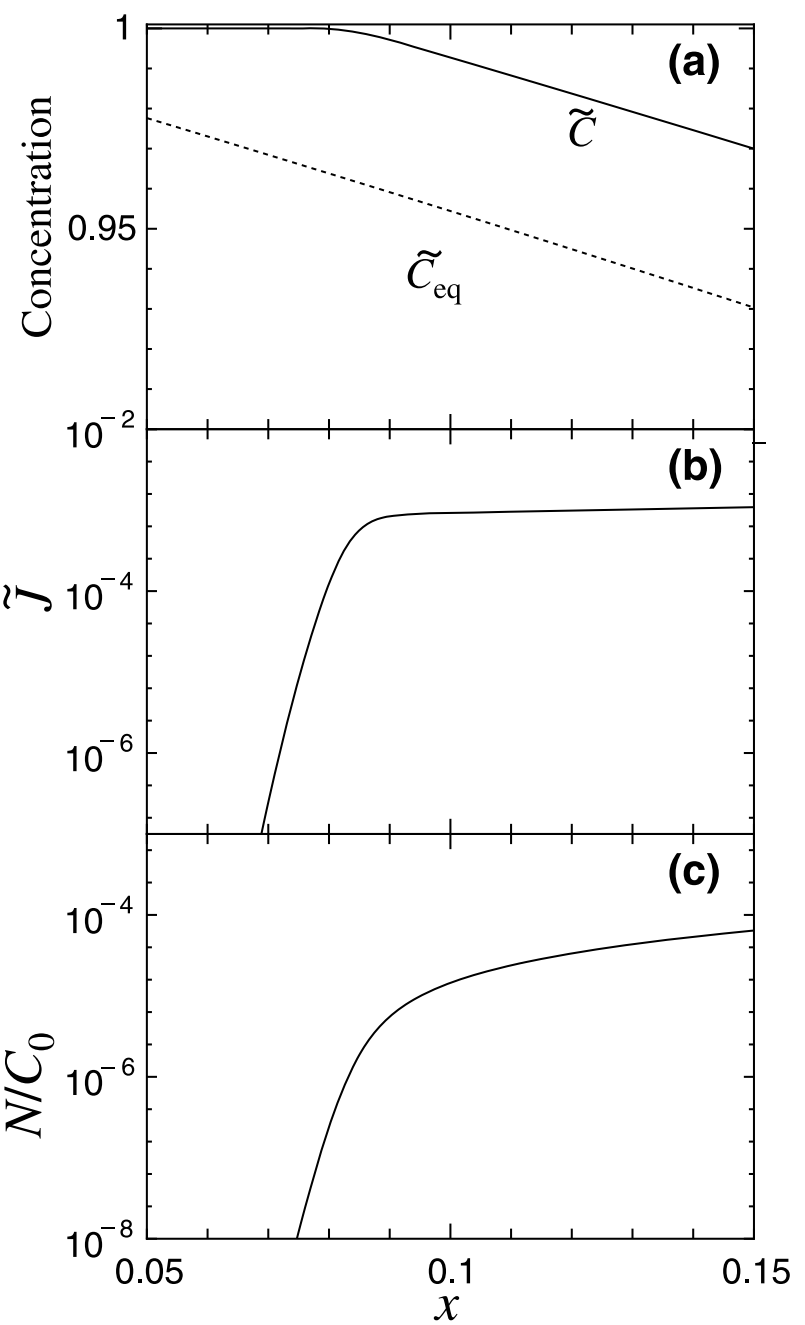

Fig. 5. Time-evolutions of the volatile concentration (a), the nucleation rate (b), and the number density of bubbles (c) for $\Theta=1 \times 10^{2}$. This corresponds to the high viscosity system. Other parameters are same as reference values. In Panel (a), the solid and dotted lines indicate the volatile concentration $C$ and the equilibrium value $C_{\mathrm{eq}}$, respectively. The volatile concentration begins to decrease around $x=0.09$. However, the nucleation rate slowly increases and the number density of bubbles also increases with time.

to the large size, they lead to the considerable decrease in the volatile concentration. As a result, the nucleation rate tends to zero and bubble nucleation stops.

\subsection{The Ostwald ripening}

Let us consider how the liquid viscosity influences the bubble growth. Time-evolution of the radius $\tilde{R}$ of a bubble nucleated at $x^{\prime}=5.5 \times 10^{-2}$ is presented in Fig. 7(a), (b), and (c) for the cases of $\Theta=1 \times 10^{7}, 2 \times 10^{4}$, and $1 \times 10^{2}$, respectively. As shown in Fig. 7(a), bubble can grow to the large size rapidly in liquid with the low viscosity. As indicated by many theoretical studies (Proussevitch et al., 1993; Toramaru, 1995; Proussevitch and Sahagian, 1998; Yamada et al., 2005), the bubble radius evolves as $R \propto t^{1 / 2}$ in this case. This means that the bubble growth is controlled by the diffusion flux of monomer. On the other hand, as found in Fig. 7(b), the liquid viscosity delays the start of the bubble growth for long time. After the delay time, bubbles expand with decompression because of the 


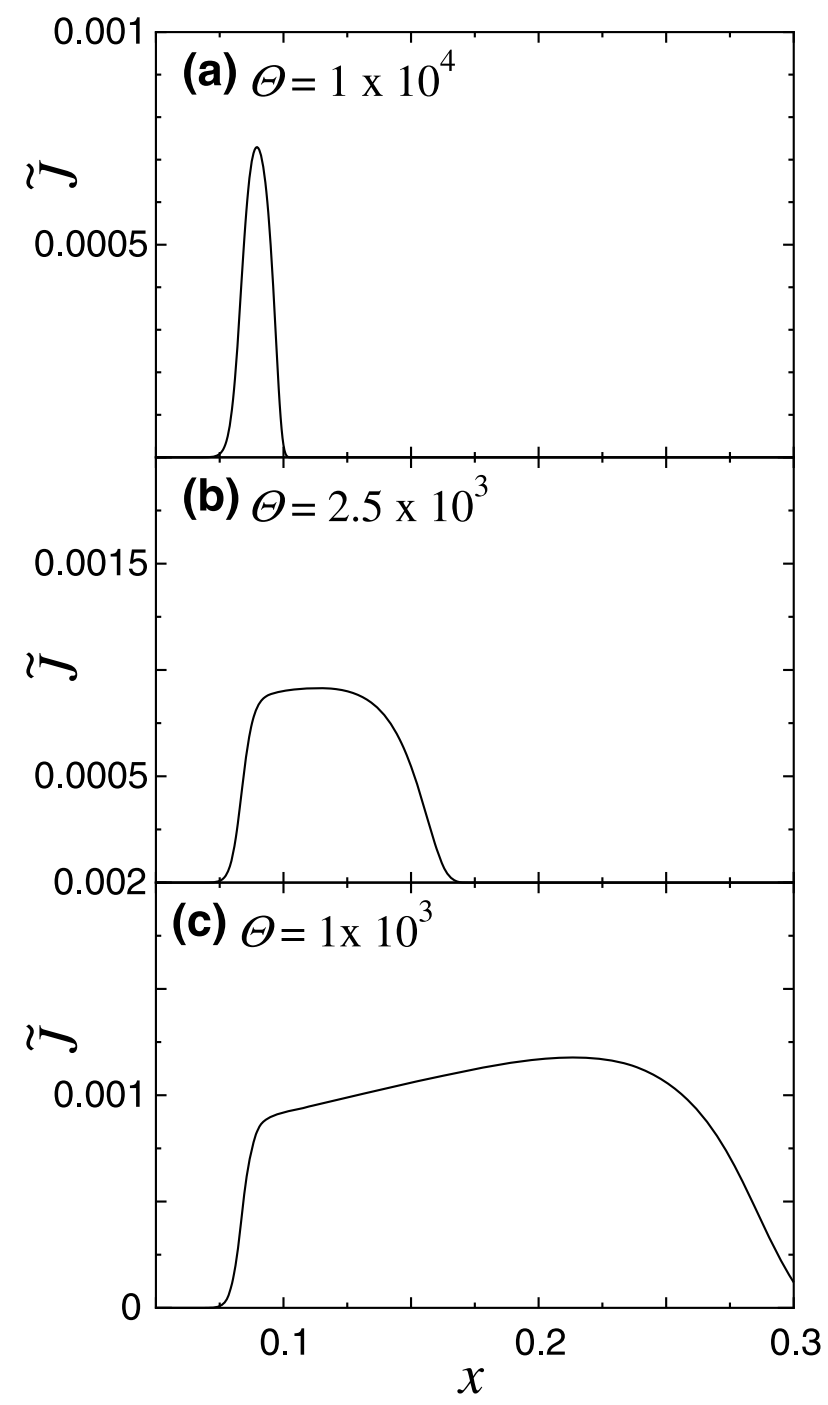

Fig. 6. Time-evolutions of the nucleation rate for $\Theta=1 \times 10^{4}$ (a), $2.5 \times 10^{3}(\mathrm{~b})$, and $1 \times 10^{3}(\mathrm{c})$. Other parameters are same as reference values. Panels (a), (b), and (c) correspond to the low, medium, and high viscosity systems in region II which is explained in Subsection 3.3, respectively. As smaller $\Theta$ (larger the viscosity), longer the duration time of nucleation.

large pressure difference between liquid and gas within a bubble. As bubbles grow enough, the pressure difference between liquid and a bubble becomes small. Then, the bubble growth becomes the diffusive growth. It is found from Panels (b) and (c) that the liquid viscosity has a significant effect on the delay time of the bubble growth. As indicated in Fig. 7(c), extreme high viscous liquid prohibits the bubble growth. According to which parameter does control the bubble growth, the behavior of growth is different. Our results agree with the behavior of the bubble growth presented in Toramaru (1995).

The dissolution of bubbles, that is, the Ostwald ripening, occurs when the bubble radius becomes smaller than the critical radius. Time-evolutions of the radius of bubbles nucleated at $x=5.5 \times 10^{-2}, 6.5 \times 10^{-2}$, and $7.8 \times 10^{-2}$ are shown in Fig. 8 for the case of $\Theta=2 \times 10^{4}$. As found in Fig. 8, bubbles nucleated earlier can grow to the larger size and they make a major contribution to the decrease

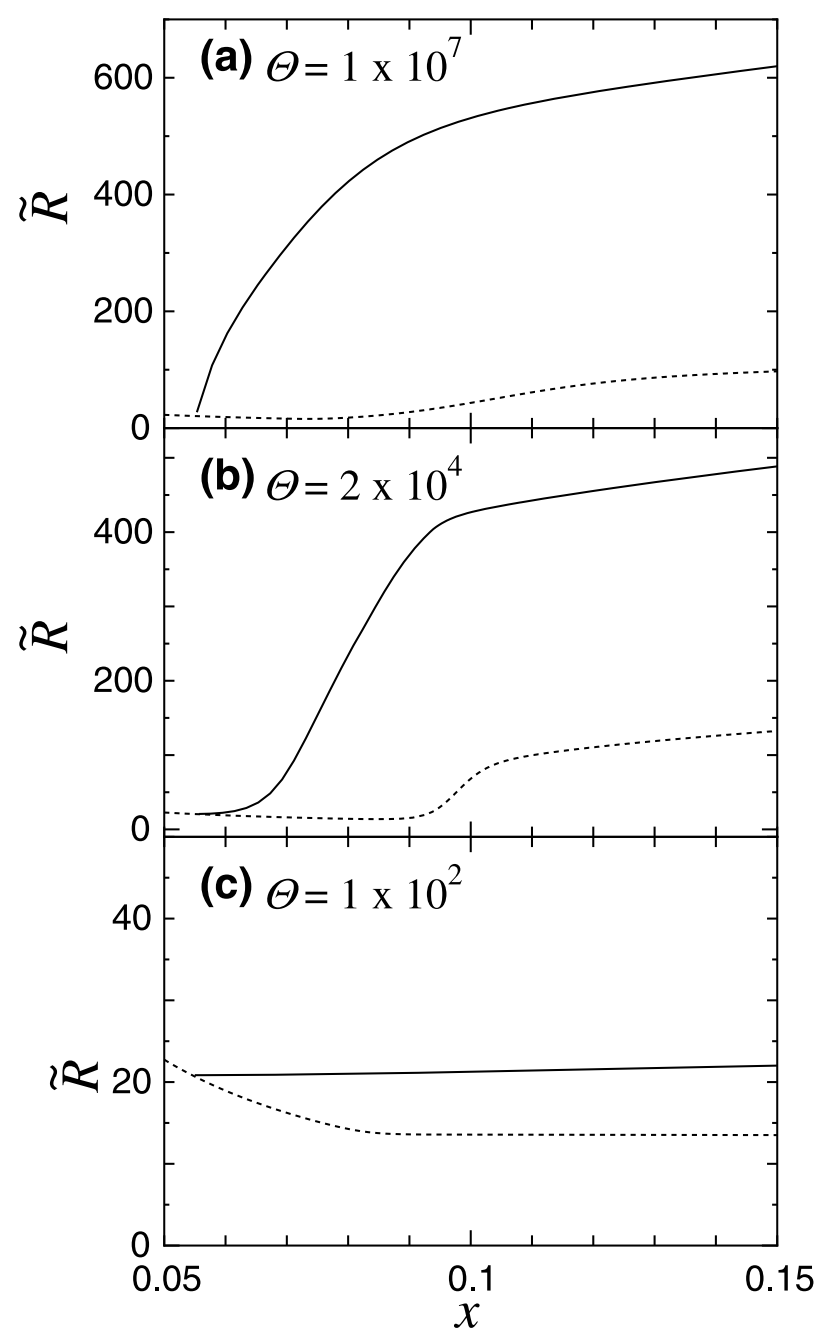

Fig. 7. Time-evolutions of the radius of bubbles for $\Theta=1 \times 10^{7}$ (a), $2 \times 10^{4}(\mathrm{~b})$, and $1 \times 10^{2}(\mathrm{c})$, respectively. Panels (a), (b), and (c) correspond to the low, medium, and high viscosity systems, respectively. Other parameters are same as reference values. The solid and dotted lines indicate the radius of bubbles nucleated at $x^{\prime}=5.5 \times 10^{-2}$ and the critical radius, respectively. In liquid with the high viscosity, the bubble growth is restrained strongly. By exhibiting the growth of bubbles nucleated at $x^{\prime}=5.5 \times 10^{-2}$, one can clearly understand the effect of the liquid viscosity on the bubble growth before the Ostwald ripening occurs.

in the volatile concentration. In other words, the timeevolution of the supersaturation ratio is mainly governed by bubbles nucleated at the early time. On the other hand, the number density of bubbles is principally determined by small bubbles nucleated at the late time. The growth of small bubbles does not proceed very much because of the delay of growth due to the viscous resistance. The critical radius is related to the supersaturation ratio (see Eq. (5)). Then, the growth rate of smaller bubbles cannot be above the ascending rate of the critical radius and these small bubbles disappear. The Ostwald ripening occurs. The typical time-evolution of dissolved bubbles corresponds to the dot-dashed line in Fig. 8. It can be also interpreted that the dissolved bubbles are absorbed by larger bubbles through the volatile concentration. The Ostwald ripening plays the same role as bubble coalescence. 


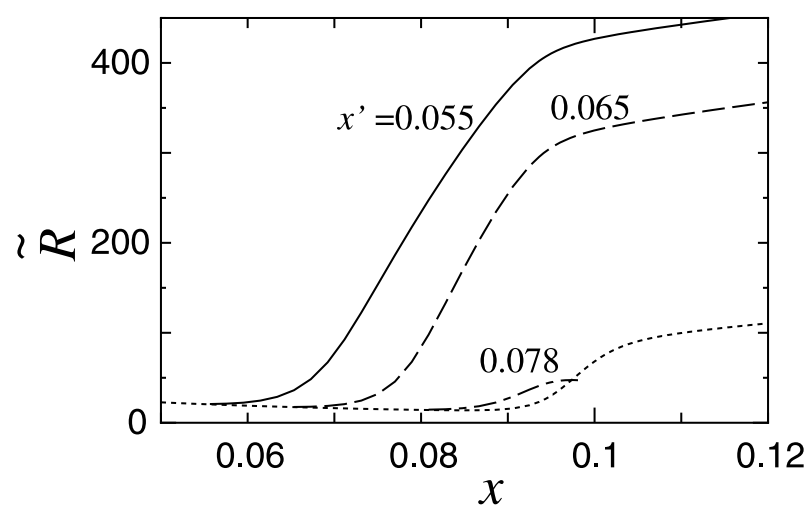

Fig. 8. Time-evolution of the radius of bubbles nucleated at different times for $\Theta=2 \times 10^{4}$. Other parameters are same as reference values. The solid, dashed, and dot-dashed lines represent the time-evolutions of the radius of bubbles nucleated at $x^{\prime}=5.5 \times 10^{-2}, 6.5 \times 10^{-2}$, and $7.8 \times 10^{-2}$, respectively. Alternatively, the dotted line corresponds to the critical radius. The size of bubbles nucleated at $x^{\prime}=7.8 \times 10^{-2}$ becomes smaller than the critical radius before $x=0.1$. Therefore, these bubbles dissolve again and disappear. Noted that bubbles with the smaller radius than the critical radius are removed from our numerical simulations. So, their time-evolutions after the Ostwald ripening are not calculated in order to save the CPU time.

\subsection{Dependency of viscosity, diffusion coefficient, and surface tension on the number density of bubbles}

Although the number density of bubbles changes with time owing to the Ostwald ripening after bubble nucleation ceases, it is helpful that we first see the maximum number density of bubbles in order to investigate the effect of the volatile diffusivity, the liquid viscosity, and the surface tension on bubble formation. The numerical results of the maximum number density are shown for $\Theta, \Lambda$, and $\tilde{\gamma}$ in Fig. 9. Panels (a) and (b) correspond to $\tilde{\gamma}=0.88$ and 2.64, respectively. As seen from Fig. 9(a) and (b), the behavior of bubble formation is divided into three regimes. Henceforth, we call each region as region I, II, and III. In the low range of $\Theta$ called region I where corresponds to the high liquid viscosity, the maximum number density does not depend on $\Theta$ but it marginally depends on $\Lambda$. On the other hand, in the high range of $\Theta$ called region III where corresponds to the low liquid viscosity, the maximum number density mostly depends on $\Lambda$. In the middle range of $\Theta$ called region II, where corresponds to the middle viscosity, the maximum number density is predominantly sensitive to $\Theta$. Regions II and III correspond to the viscosity-controlled and diffusion-controlled regime found by Toramaru (1995). On the other hand, region I is newly presented by our study. The solid and dot-dashed lines show the border lines between regions I and II and regions II and III, respectively. They are provided by the analytical expressions described later. The value of $\Theta$ on each border line between regimes is named as $\Theta_{\mathrm{I} / \mathrm{II}}$ and $\Theta_{\mathrm{II} / \mathrm{III}}$. Two dotted lines correspond to the border lines between regions I and II and regions II and III speculated from numerical results. In our study, each regime is distinguished on the basis of the analytical expressions although the analytical results diverge from the numerical ones owing to the approximation in the analytical conditions.

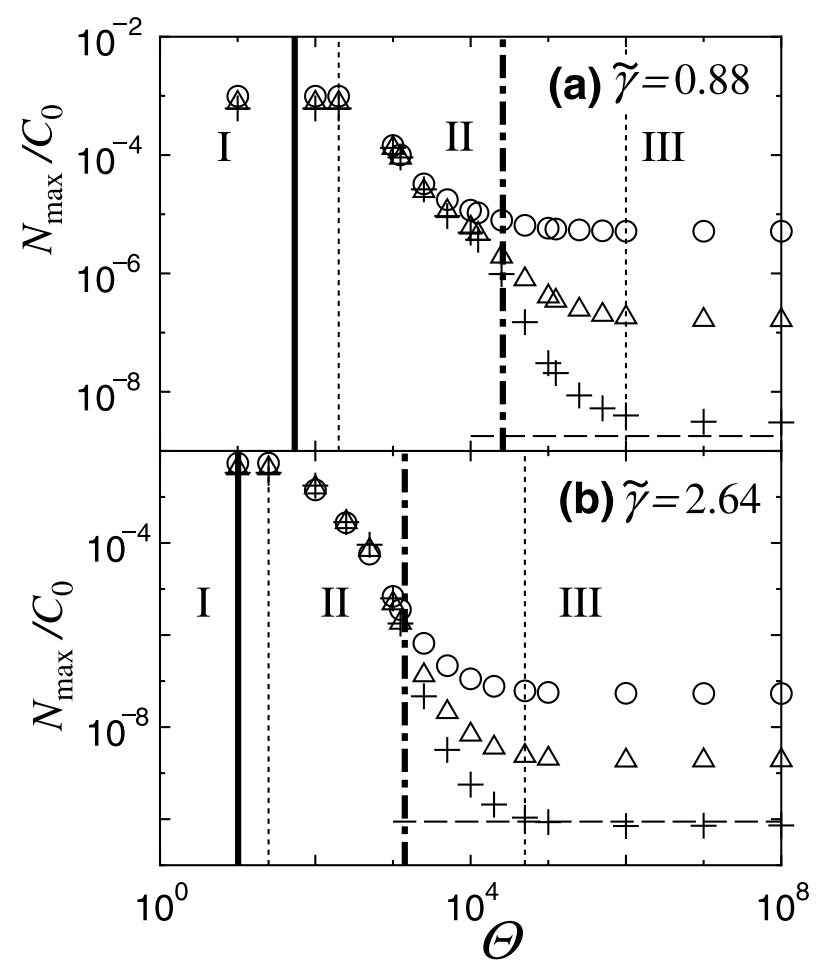

Fig. 9. The maximum number densities for $\Lambda=9 \times 10^{5}$ (circles), $9 \times 10^{6}$ (triangles), and $9 \times 10^{7}$ (crosses) are plotted as a function of $\Theta$. Panels (a) and (b) correspond to $\tilde{\gamma}=0.88$ and 2.64 , respectively. The vertical thick solid and dot-dashed lines represent the border line between regions I and II and regions II and III, respectively, in the case of $\Lambda=9 \times 10^{7}$. Noted that the values of the threshold between regions I and II and regions II and III are given by Eqs. (50) and (49), respectively, in the case of $\Lambda=9 \times 10^{7}$. On the other hand, the dotted lines between regions I and II and regions II and III are settled by the numerical results. In addition, the dashed line represents the approximate number density of bubbles given by Yamada et al. (2005), that is, Eq. (47), for $\Lambda=9 \times 10^{7}$.

In region I, the maximum number density of bubbles is constant regardless of the magnitude of the viscosity because their growth is suppressed by the viscous resistance of liquid during the decompression time. As shown in Fig. 9, the calculated maximum number densities of bubbles are $6.1 \times 10^{-4}, 7.6 \times 10^{-4}$, and $9.9 \times 10^{-4}$ in the case of $\tilde{\gamma}=0.88$ and $\Theta=1 \times 10$ for $\Lambda=9 \times 10^{7}, 9 \times 10^{6}$, and $9 \times 10^{5}$, respectively, and $3.3 \times 10^{-3}, 4.2 \times 10^{-3}$, and $5.5 \times 10^{-3}$ in the case of $\tilde{\gamma}=2.64$ and $\Theta=1 \times 10$ for $\Lambda=9 \times 10^{7}, 9 \times 10^{6}$, and $9 \times 10^{5}$, respectively. The weak dependence of maximum number density on $\Lambda$ originates from the preexponential factor of the nucleation rate (see in detail in Appendix A). Since the bubble size is almost equivalent to the critical size, the maximum number density of bubbles is given by

$$
N_{\max }^{\mathrm{I}} \simeq \frac{3 \phi}{4 \pi R_{\mathrm{cr}}^{3}},
$$

where $\phi$ is the void ratio. In our paper, the calculations are terminated at $\phi=0.3$ and the number density of bubbles reaches to the maximum value at $\phi=0.3$. Noted that the concrete analytical expression of the maximum number density in region I is minutely described in Appendix A. 


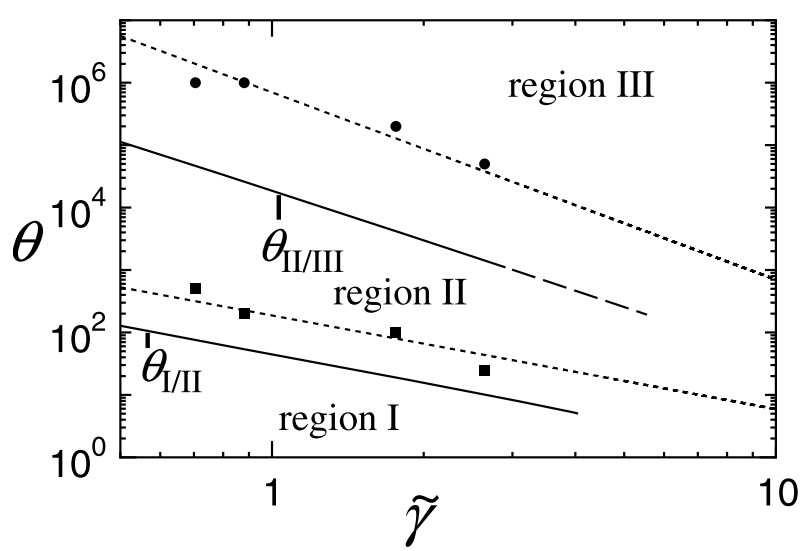

Fig. 10. The regime of the bubble formation process as function of two parameters, $\tilde{\gamma}$ and $\Theta$ for $\Lambda=9 \times 10^{7}$. Two solid lines indicate the boundary lines between regions I and II and regions II and III. The boundary line between regions II and III, which is labeled as $\Theta_{\mathrm{II} / \mathrm{III}}$, is decided by the analytical expression (Eq. (49)) and the boundary line between regions I and II, which is labeled as $\Theta_{\mathrm{I} / \mathrm{II}}$, by the analytical expression (Eq. (50)). The circles represent the values of $\Theta$ at which the maximum number density begins to vary with $\Theta$, whereas the squares correspond to the values of $\Theta$ from which the mamimun number density is constant against $\Theta$. These are predicted on the basis of numerical results. Two dotted lines are lines optimized from data of circles or squares. The gradient of each dotted line is given on the basis of the analytical expression. The dotted line through circles is given as $\log _{10} \Theta=-3 \log _{10} \tilde{\gamma}+5.8$ and the dotted line through squares as $\log _{10} \Theta=-1.5 \log _{10} \tilde{\gamma}+2.3$. The dashed part of $\Theta_{\mathrm{II} / \mathrm{III}}$ indicates that the assumption of small $x_{J}$, which is used in deriving Eq. (49), is rough and the analytical expression may be inappropriate.

In region II, the maximum number densities depend on only $\Theta$. In this regime, the maximum number density dramatically decreases as $\Theta$ increases. In region II, the dependence only on $\Theta$ is caused from the fact that the viscosity has a significant effect on the bubble growth (see Fig. 7(b)). On the other hand, in region III, the maximum number density changes as $N_{\max } \propto \Lambda^{-3 / 2}$ and this dependence is caused from the fact that the bubble growth is controlled by the diffusion and reproduced by the approximate solution in Yamada et al. (2005). In addition, there is a transition area where the maximum number density of bubbles changes according to not only $\Lambda$ but also $\Theta$ before region II. Such dependence of the bubble number density on the viscosity and the volatile diffusivity is qualitatively indicated by Toramaru (1995).

As compared with Fig. 9(a), the range of region III in Fig. 9(b) is wider for a given $\Lambda$. In other words, region III shifts to the lower range of $\Theta$ as the surface tension is larger. The dependence of the area of each regime on $\tilde{\gamma}$, which is presumed as the surface tension, is demonstrated in Fig. 10 for $\Lambda=9 \times 10^{7}$. As found in Fig. 10, the values of $\Theta_{\mathrm{I} / \mathrm{II}}$ and $\Theta_{\mathrm{II} / \mathrm{III}}$ decrease by about a factor of 5 and an order of 10 , respectively, as $\tilde{\gamma}$ increases threefold. This means that the area of region II is narrower as the larger $\tilde{\gamma}$. Bubble formation is basically controlled by the viscosity in the smaller range of $\Theta$ than $\Theta_{\mathrm{II} / \mathrm{III}}$, whereas it is mainly controlled by the diffusion in the larger range of $\Theta$ than $\Theta_{\mathrm{II} / \mathrm{III}}$. Actually, as found from numerical results (see in Fig. 9), the influence of the viscosity on the nucleation process of bubbles

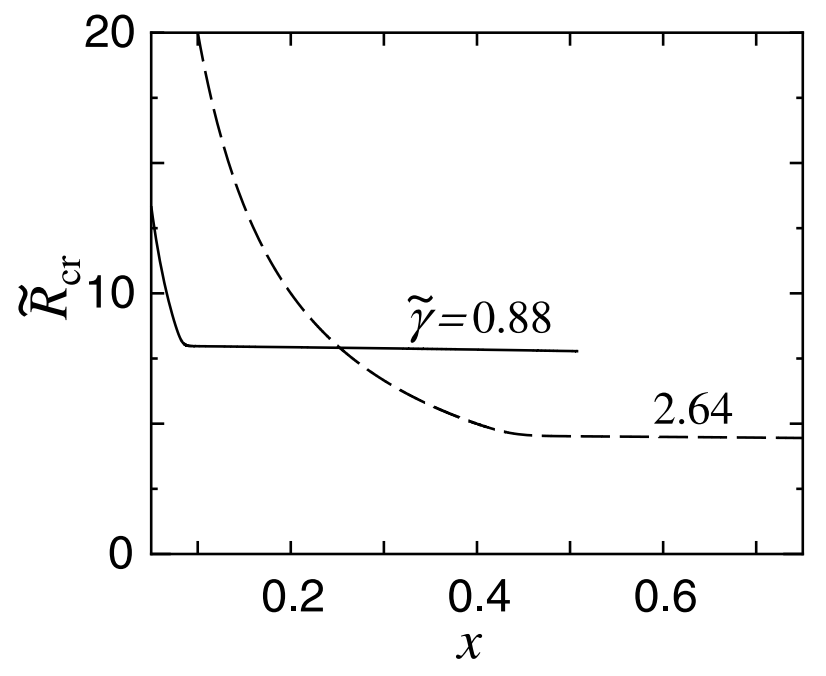

Fig. 11. Time-evolutions of the critical radius in the case of $\tilde{\gamma}=0.88$ (solid line) and 2.64 (dashed line). The parameter $\Theta$ is $1 \times 10$ and other parameters are equal to the reference values. In the case of $\tilde{\gamma}=0.88$, the numerical simulation ends since the void ratio becomes 0.3 at $x \simeq 0.5$. Thus, the solid line terminates there.

gradually appears from the smaller range of $\Theta$ in region III. Consequently, the maximum number density depends on $\Lambda$

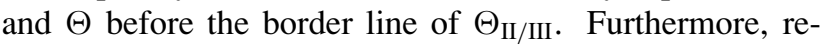
gion I also begins from the larger range of $\Theta$ than $\Theta_{\mathrm{I} / \mathrm{II}}$. In Fig. 10, two dotted lines are numerically determined. It is, then, noted that the gradient of lines is given by a simple form of analytical expressions, as described later.

All values of parameters used in this study do not cover the range of realistic magma parameters. Especially, the surface tension is smaller than that for water vapor and silicic melt interface, which is approximately $0.1 \mathrm{~N} / \mathrm{m}$ according to Bagdassarov et al. (2000). Given the realistic value of the surface tension, bubble formation is expected to proceed in a similar way as that in region III for any viscosity of silicic melts at the high temperature (Hess and Dingwell, 1996; Zhang et al., 2007).

The larger surface tension suppresses bubble nucleation. It is found from Fig. 9(b) that the number density of nucleated bubbles is small compared to that in Fig. 9(a) in region III for a given $\Lambda$. When the number density of bubbles is smaller, each bubble can grow larger. On the other hand, the number density of bubbles is larger in region $I$ in the case of Panel (b) than Panel (a) for a given $\Lambda$. The larger supersaturation ratio is needed to trigger the nucleation in the case of the larger surface tension. This implies that the critical radius is smaller. Therefore, the number density of bubbles increases in region I. Figure 11 shows the time-evolutions of the critical radius in the case of $\tilde{\gamma}=0.88$ and 2.64. The parameter $\Theta$ is $1 \times 10$ and other parameters are set to be the reference values. As seen in this figure, the critical radius actually settles down at the lower level in the case of the larger surface tension.

The Ostwald ripening leads to the decrease in the number density of bubbles. So, after bubble nucleation ceases, the number density of bubbles may extensively decrease from the maximum number density of bubbles. We call 


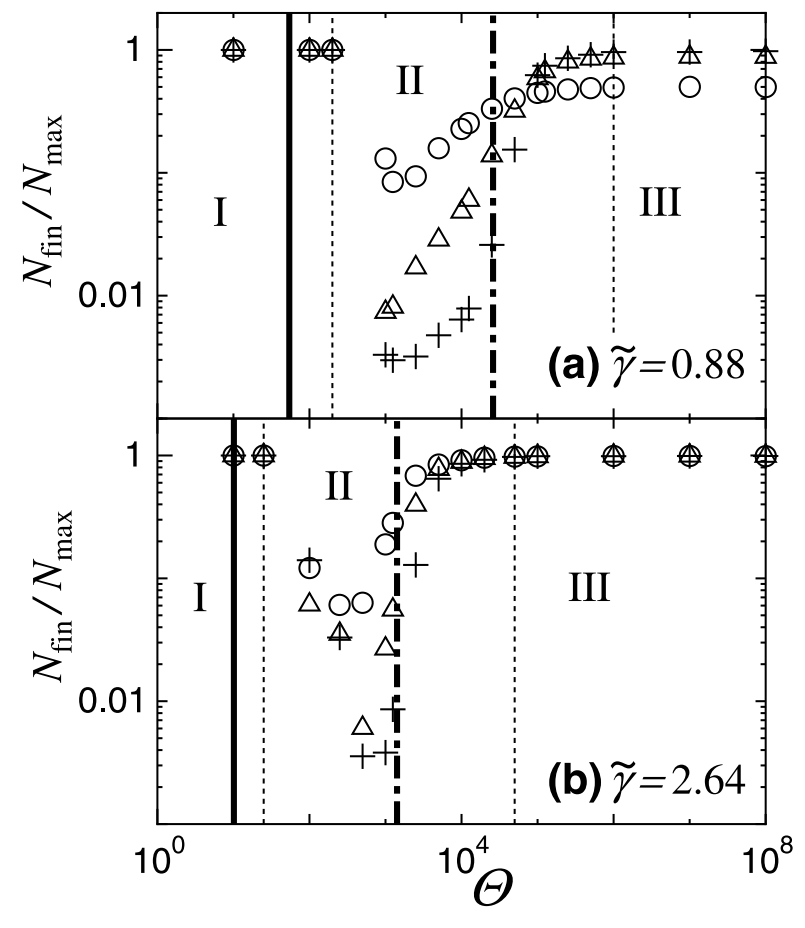

Fig. 12. The ratio of the maximum number density of bubbles to the final number density of bubbles $N_{\text {fin }} / N_{\max }$ for $\Lambda=9 \times 10^{5}$ (circles), $9 \times 10^{6}$ (triangles), and $9 \times 10^{7}$ (crosses) are plotted as a function of $\Theta$. Panels (a) and (b) correspond to $\tilde{\gamma}=0.88$ and 2.64 , respectively. The vertical solid and dot-dashed lines represent the border line between regions I and II and regions II and III in the case of $\Lambda=9 \times 10^{7}$, respectively. The dotted lines correspond to boundaries between each regime determined from numerical results.

the number density of bubbles obtained at the end of our numerical simulations the final number density of bubbles. The ratios of the final number density of bubbles $N_{\text {fin }}$ to the maximum number density of bubbles $N_{\max }$ are represented for $\Theta, \Lambda$, and $\tilde{\gamma}$ in Fig. 12. Then, the values of $\tilde{\gamma}$ are 0.88 and 2.64 in Panels (a) and (b), respectively. It can be seen from Fig. 12 that the ratio $N_{\text {fin }} / N_{\max }$ is equivalent to unity in region I and the final number density of bubbles slightly diminishes from the maximum number density in region III. In region II, the ratio decreases greatly. For example, it falls below $1 \times 10^{-2}$ in both Panels (a) and (b) in the case of $\Lambda=9 \times 10^{7}$. Smaller $N_{\text {fin }} / N_{\max }$ is brought as larger the diffusion coefficient. When the diffusion coefficient is large, bubbles nucleated at the early time can rapidly gather many volatile molecules in liquid. From our numerical results, it is concluded that almost all of nucleated bubbles can survive in regions I and III. On the other hand, in region II, small bubbles nucleated at the late time disappear due to the Ostwald ripening.

One can wonder about the dependency of the surface tension, the diffusion coefficient, and the viscosity on the $N_{\max }$ to $N_{\text {fin }}$ ratio. For example, when the surface tension is large, region III extends toward the range of lower $\Theta$ and the drop rate of $N_{\text {fin }} / N_{\text {max }}$ appears to be very steep, whereas the minimum values of $N_{\text {fin }} / N_{\text {max }}$ may be insensitive to the surface tension. It is, however, difficult to analytically clarify the relation between the ratio and the material parameters such as the diffusion coefficient, the viscosity, and the surface

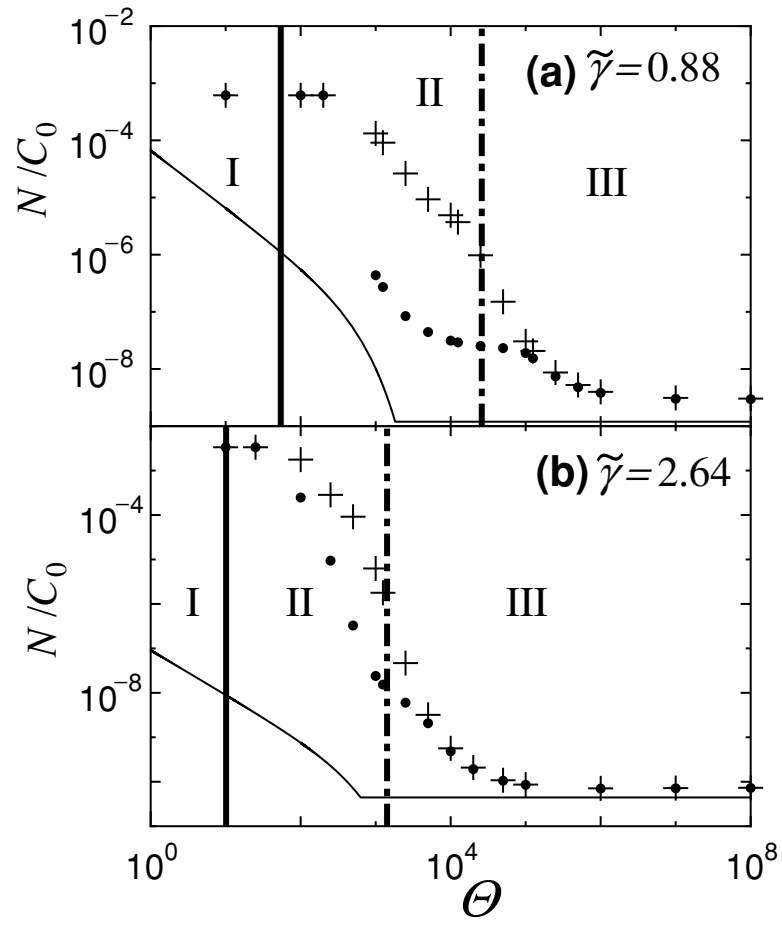

Fig. 13. The number densities against $\Theta$ in the case of $\tilde{\gamma}=0.88$ (a) and 2.64 (b) for $\Lambda=9 \times 10^{7}$. The cross, the circle, and the solid line correspond to the maximum number density of bubbles, $N_{\max }$, the final number density of bubbles, $N_{\text {fin }}$, and the results presented by Toramaru (1995), respectively. In the large range of $\Theta$, results of both models are consistent within one order of the magnitude. The results of Toramaru (1995) are wholly much smaller than $N_{\max }$ and somewhat smaller than $N_{\text {fin. }}$.

tension. Our study is in progress to determine which range of parameters leads to the large decrease in bubble number density due to the Ostwald ripening.

Toramaru (1995) presented the viscosity and diffusion controlled regime, which correspond to regions II and III in our paper, respectively, for the nucleation and growth processes and derived the empirical expression of the final number density of bubbles from his numerical results. The maximum number density of bubbles, the final number density of bubbles, and the results presented by Toramaru (1995) are shown in Fig. 13(a) and (b) in the case of $\tilde{\gamma}=0.88$ and 2.64 , respectively, for $\Lambda=9 \times 10^{7}$. As seen in Fig. 13, our results are greater than ones by Toramaru's empirical expression and begin to increase at the larger $\Theta$ than Toramaru's result. The size distribution of bubbles is taken into account in our study. On the other hand, Toramaru (1995) assumed all bubbles to have an equal size. The number density of bubbles is basically governed by small bubbles nucleated at the late time. The growth process of small bubbles is easy to be influenced by the viscosity. Hence, the effect of the size distribution yields the discrepancy in the behavior of the bubble number density between both studies.

\subsection{Analytical expression for boundary between re- gions II and III}

We consider how large viscosity the transition from regions II to III occur at. On the border line between regions 
II and III, the influence of the diffusivity and the viscosity upon bubble formation switches. So, this border line is the important line. Each region is basically defined by the behavior of the maximum number density of bubbles. Hence, an analytical expression of the maximum bubble number density is necessary to see its dependence on material quantities. In order to do so, let us derive approximate solutions to the maximum number density of bubbles in the case of region II in the same manner used in Yamada et al. (2005).

In the analytic procedure, we assume that nondimensional time $x$ and $|1-\tilde{C}|$ are small as compared with unity. Therefore, we assume that $\tilde{C}$ can be replaced with unity on the right hand side of basic equations. Hereafter, let us call this assumption as the zero-th order approximation. The non-dimensional equilibrium concentration is given in the zero-th order approximation by

$$
\tilde{C}_{\mathrm{eq}}=1-(1-\beta) \frac{x}{m}
$$

where the second and higher order terms of $x$ are neglected. Then, the non-dimensional form of the zero-th order nucleation rate $\tilde{J}_{0}$ is

$$
\tilde{J}_{0}(x)=\frac{3 \alpha \Lambda}{8 m \sqrt{\pi^{3} \tilde{\gamma}}}(1-\beta) x \exp \left[-\frac{a^{2}}{x^{2}}\right]
$$

with

$$
a=2\left(\frac{\tilde{\gamma}}{3}\right)^{3 / 2}(1-\beta)^{-1}
$$

The expression of the bubble radius $\tilde{R}\left(x, x^{\prime}\right)$ is required to evaluate the time-evolution of the volatile concentration $\tilde{C}(x)$. To derive the approximate expression of the bubble radius, the interfacial concentration is set to be the initial concentration because the internal pressure is high for a small bubble in the case of the high viscosity. Replacing $\tilde{C}_{\text {in }}$ with unity in Eq. (2), the internal pressure $\tilde{P}_{\text {gas }}$ is given as $1-\beta x$. Integrating Eq. (22) in the zero-th order approximation, we have (see Appendix B in detail)

$$
\tilde{R}\left(x, x^{\prime}\right)=\tilde{R}^{\prime}\left(x, x^{\prime}\right) \exp \left[\frac{b^{2}}{3}\left(x^{2}-x^{\prime 2}\right)\right],
$$

where $\tilde{R}^{\prime}\left(x, x^{\prime}\right)$ is a function of $x$ and $x^{\prime}$ and is defined by

$$
\begin{aligned}
\tilde{R}^{\prime}\left(x, x^{\prime}\right)= & \frac{2 \tilde{\gamma}}{3(1-\beta) x^{\prime}}-\frac{\tilde{\gamma}}{3} \sqrt{\frac{\pi \Theta}{2(1-\beta)}} \\
& \cdot\left[\operatorname{erf}\left(\frac{b x}{\sqrt{3}}\right)-\operatorname{erf}\left(\frac{b x^{\prime}}{\sqrt{3}}\right)\right] e^{b^{2} x^{\prime 2} / 3}
\end{aligned}
$$

and $b$ is given by

$$
b=\sqrt{\frac{3(1-\beta) \Theta}{8}} .
$$

As seen from Eq. (38), bubbles exponentially expand when the growth is controlled by the viscosity.

Substituting Eqs. (36) and (38) into Eq. (23), it is, in the zero-th order approximation, written as

$$
\begin{aligned}
1-\tilde{C}(x)= & \frac{3 \alpha \Lambda}{8 m \sqrt{\pi^{3} \tilde{\gamma}}}(1-\beta) e^{b^{2} x^{2}} \int_{0}^{x} x^{\prime} \tilde{R}^{\prime}\left(x, x^{\prime}\right)^{3} \\
& \cdot \exp \left[-b^{2} x^{\prime 2}-\frac{a^{2}}{x^{\prime 2}}\right] \mathrm{d} x^{\prime}
\end{aligned}
$$

The exponential function in the integrand has a maximum at $x^{\prime}=\sqrt{a / b}$. Henceforth we define $\sqrt{a / b}$ as $x_{0}$. This implies that bubbles nucleated around $x=x_{0}$ govern the total volume of bubbles.

Because of the strong sensitivity of the exponential factor to $x^{\prime}$, we can expand the exponential factor in the integrand around $x^{\prime}=x_{0}$. Performing the integration, we obtain the volatile concentration as

$$
\begin{aligned}
\tilde{C}(x)= & 1-\frac{\alpha \Lambda}{2 \pi m}\left(\frac{\tilde{\gamma}}{18(1-\beta) \Theta^{3}}\right)^{1 / 4} \tilde{R}^{\prime}\left(x, x_{0}\right)^{3} \\
& \cdot \exp \left[b^{2} x^{2}-2 a b\right],
\end{aligned}
$$

where we assumed that $4 b^{2} \gg 1$. For $\Theta=2 \times 10^{4}, b$ is estimated as 81 . The non-dimensional time $x_{J}$ when the nucleation rate has its maximum is given by the solution $\mathrm{d} S / \mathrm{d} x=0$. Using Eqs. (3) and (42), we have the following implicit equation for $x_{J}$ as

$$
\begin{aligned}
\tilde{R}^{\prime}\left(x_{J}, x_{0}\right)^{3} b x_{J} e^{b^{2} x_{J}^{2}}= & 2^{7 / 4} \pi \alpha^{-1}(1-\beta)^{3 / 4} \tilde{\gamma}^{-1 / 4} \Lambda^{-1} \\
& \cdot \Theta^{1 / 4} e^{2 a b}
\end{aligned}
$$

For parameters in Fig. 4, the non-dimensional time $x_{J}$ is $7.9 \times 10^{-2}$, which is slightly small compared with that in Fig. 4. Noted that the derivation of Eq. (43) is described in detail in Appendix C.

Because of the exponential dependence of the nucleation rate on $x$, it can be expanded around $x=x_{J}$. Note that this expansion is valid only when the nucleation rate has a sharp maximum like Fig. 6(a). Then, using Eq. (42), the approximate expression of the nucleation rate is given by

$$
\tilde{J}=\tilde{J}_{\max } \exp \left[-\frac{2 a^{2} b^{2}}{x_{J}^{2}}\left(x-x_{J}\right)^{2}\right],
$$

where $\tilde{J}_{\max }$ is the maximum nucleation rate given by

$$
\tilde{J}_{\max }=\frac{3 \alpha \Lambda}{8 m \sqrt{\pi^{3} \tilde{\gamma}}}(1-\beta) x_{J} \exp \left[-\left(\frac{a}{x_{J}}\right)^{2}-\left(\frac{x_{0}}{x_{J}}\right)^{4}\right] \text {. }
$$

Substituting Eq. (44) into Eq. (31), we have for the maximum number density of bubbles

$$
\begin{aligned}
\frac{N_{\max }^{\mathrm{II}}}{C_{0}}= & \frac{\alpha \Lambda}{6 \pi m} \sqrt{\frac{\tilde{\gamma}^{2}}{(1-\beta) \Theta}}\left(\frac{a}{x_{J}}\right)^{-2} \\
& \cdot \exp \left[-\left(\frac{a}{x_{J}}\right)^{2}-\left(\frac{x_{0}}{x_{J}}\right)^{4}\right] .
\end{aligned}
$$

Substituting the values used in Fig. 4 into Eq. (46), the approximate maximum number density is $2.4 \times 10^{-6}$, whereas the numerical value is $1.6 \times 10^{-6}$. Noted that the difference between Eq. (46) and numerical results increases with the liquid viscosity (decreasing $\Theta$ ). In the case of smaller $\Theta$, the nucleation rate becomes a trapezoid form like Fig. 6(b) and (c). In addition, the assumption that $|1-\tilde{C}| \ll 1$ is wrong. Therefore, although Eq. (46) cannot be acceptable in the whole range of region II, it is still valid in the vicinity of the transition from regions II to III. 
According to Yamada et al. (2005), the approximate maximum number density in region III is given by

$$
\frac{N_{\max }^{\mathrm{III}}}{C_{0}}=\frac{3 \alpha \Lambda}{16 e \pi m} \sqrt{\frac{2}{\tilde{\gamma}}}(1-\beta) \frac{x_{J}^{4}}{a^{2}} \exp \left[-\left(\frac{a}{x_{J}}\right)^{2}\right],
$$

where $x_{J}$ is given by

$$
\begin{aligned}
\frac{x_{J}^{7}}{a^{7}} \exp \left(-\frac{a^{2}}{x_{J}^{2}}\right)= & 2 \pi\left(\frac{m}{3}\right)^{3 / 2} \alpha^{-5 / 2}(1-\beta)^{5 / 2} \\
& \cdot \Lambda^{-5 / 2}\left(\frac{\tilde{\gamma}}{3}\right)^{-11 / 2} .
\end{aligned}
$$

The value of the threshold $\Theta_{\mathrm{II} / \mathrm{III}}$ is determined from the condition that the maximum number density of bubbles in region II is equal to that in region III. Although the maximum number density diverges from $N_{\max }^{\mathrm{III}}$ in the proximity of $\Theta_{\mathrm{II} / \mathrm{III}}$, the bubble formation process in region III are mostly governed by the diffusive growth of bubbles. Therefore, it is still available to determine analytically the value of $\Theta_{\mathrm{II} / \mathrm{III}}$. Equating Eq. (46) to Eq. (47), the threshold of the transition from regions II to III is given by (see Appendix D in detail)

$$
\Theta_{\mathrm{II} / \mathrm{III}}=\frac{5}{(1-\beta)} \frac{a^{2}}{x_{J}^{4}} .
$$

Putting reference values other than $\Theta$ into this equation, $\Theta_{\text {II/III }}$ is approximately estimated as $3 \times 10^{4}$. In Fig. 9, the vertical dot-dashed line represents the value of $\Theta_{\text {II } / \mathrm{III}}$. Yamada et al. (2005) obtained $a^{2} / x_{J}^{2}=2.5 \ln \Lambda+\ln B_{1}$ as an approximate estimation of Eq. (48), where $B_{1}$ is $\alpha^{2.5}(\tilde{\gamma} / 3)^{5.5}(3 / m)^{1.5} / 2 \pi /(1-\beta)^{2.5}$. Substituting this approximate evaluation into Eq. (49), we find that the parameter $\Theta_{\text {II/III }}$ logarithmically depends on the diffusion coefficient and inversely on the surface tension through $a^{2}$ $\left(\propto \tilde{\gamma}^{3}\right)$.

In Eq. (38), replacing $x^{\prime}$ with $x_{J}$ and since $x$ is comparable to $x_{J}$ immediately after the nucleation, the exponent of the exponential function is approximately given by $2 b^{2} x_{J}\left(x-x_{J}\right) / 3$. Thus, the expansion time of bubbles is typically given as $3 t_{P} / 2 b^{2} x_{J}$. On the other hand, $\left(\sqrt{2} x_{J}^{3} / a^{2}\right) t_{P}$ corresponds to the duration time of nucleation in the case that the bubble growth is governed by the diffusivity (see Yamada et al. (2005)). Hence, Eq. (49) implies that the effect of the viscosity on bubble formation is significant when the expansion time of bubbles is longer than about 0.6 times duration time of nucleation.

\subsection{Analytical expression for boundary between re- gions I and II}

Let us derive an analytical expression of $\Theta_{\mathrm{I} / \mathrm{II}}$. In region I, the bubble growth does not proceed much owing to the viscous resistance. In other words, region I is characterized by the fact that the typical growth time of bubbles is longer than the decompression time. As mentioned previously, the typical growth time of bubbles, which is controlled by the viscosity, is roughly estimated as $3 t_{P} / 2 b^{2} x$. Therefore, since the typical growth time is equivalent to the decompression time at the boundary between regions I and II, we obtain $t_{P}=3 t_{P} / 2 b^{2} x$.

We now consider the characteristic value of $x$ in region I such as $x_{J}$ described above. In region $\mathrm{I}$, the nucleation rate continues to increase. As described in Appendix A, there is a turning point that the nucleation rate changes from the rapid increase to the slow increase (see in Fig. 5). The time, $x_{1}$, corresponding to the turning point is evaluated as the analytical form in Appendix A. We regard the growth time of bubbles nucleated at $x^{\prime}=x_{1}$ as a characteristic growth time of bubbles in region I. Accordingly, replacing $x$ with $x_{1}$ and using Eq. (40), we have

$$
\Theta_{\mathrm{I} / \mathrm{II}}=\frac{4}{(1-\beta) x_{1}},
$$

where $x_{1}$ is given by Eq. (A.4). In order to understand the relation between $\Theta_{\mathrm{I} / \mathrm{II}}$ and each parameter, we consider an analytical expression of $x_{1}$ assuming that $\beta x_{1} \ll 1$. Under this assumption, $x_{1}$ is approximately given by

$$
x_{1}^{2} \exp \left(\frac{a^{2}}{x_{1}^{2}}\right)=\frac{\alpha \Lambda \tilde{\gamma}^{2.5}}{9 \pi^{1.5}(1-\beta)^{3}} .
$$

The variation of the preexponential function on the left hand side of Eq. (51) is less drastic than that of the exponential function. Hence, we obtain the first-order expression of $x_{1}$ as $x_{1}=a / \sqrt{\ln \Lambda+\ln B_{2}}$, where $B_{2}$ is $\alpha \tilde{\gamma}^{2.5} / 9 \pi^{1.5}(1-\beta)^{3}$. In addition, a second-order approximation of $x_{1}$ is given by

$$
x_{1}=\frac{a}{\sqrt{\ln \Lambda+\ln B_{2}}}\left[1+\frac{\ln a-0.5 \ln \left\{\ln \left(\Lambda B_{2}\right)\right\}}{\ln \left(\Lambda B_{2}\right)}\right] .
$$

Substituting Eq. (52) into Eq. (50), it is found that $\Theta_{\mathrm{I} / \mathrm{II}}$ is sensitive to logarithmically $\Lambda$ and inversely $\tilde{\gamma}^{3 / 2}$ through $a$.

\subsection{The size distribution of bubbles}

In this subsection, we see the time-evolution of the cumulative number density of bubbles. In Fig. 14, the cumulative size distributions are provided for the case of $\Theta=1 \times 10^{7}$ (a), $2 \times 10^{4}$ (b), and $1 \times 10^{2}$ (c), respectively. In Panel (a), the cumulative number densities of bubbles at $x=0.08,0.15$, and 0.3 are presented. It is shown in this figure that most of bubbles are distributed to a relatively narrow size range. When the bubble growth is controlled by the diffusivity, the growth rate of small bubbles is larger than that of large bubbles. Thus, the dispersion in the bubble size distribution becomes smaller as bubbles grow larger. Of course, such unimodal size distribution means that most bubbles have similar size.

In Panel (b), the solid, dotted, and dashed lines correspond to the cumulative number density of bubbles at $x=$ $0.09,0.15$, and 0.3 , respectively. It is seen from Fig. 14(b) that the cumulative number density of bubbles just after the nucleation stops (at $x=0.09$ ) has a broader distribution than that in Fig. 14(a). As mentioned previously, the growth rate of bubbles immediately after the nucleation is approximately given by $3 / 2 b^{2} x_{J}$. This sole growth rate means that all bubbles grow at the almost equal rate. Hence, such shape as this figure causes. On the other hand, when bubbles grow largely, the growth law of bubbles shifts from the exponential growth to the diffusive growth because the viscosity has little effect on large bubbles. So, the size distribution of bubbles gradually resembles that of Fig. 14(a) in the shape. However, the size distribution in Fig. 14(b) has yet a wider 


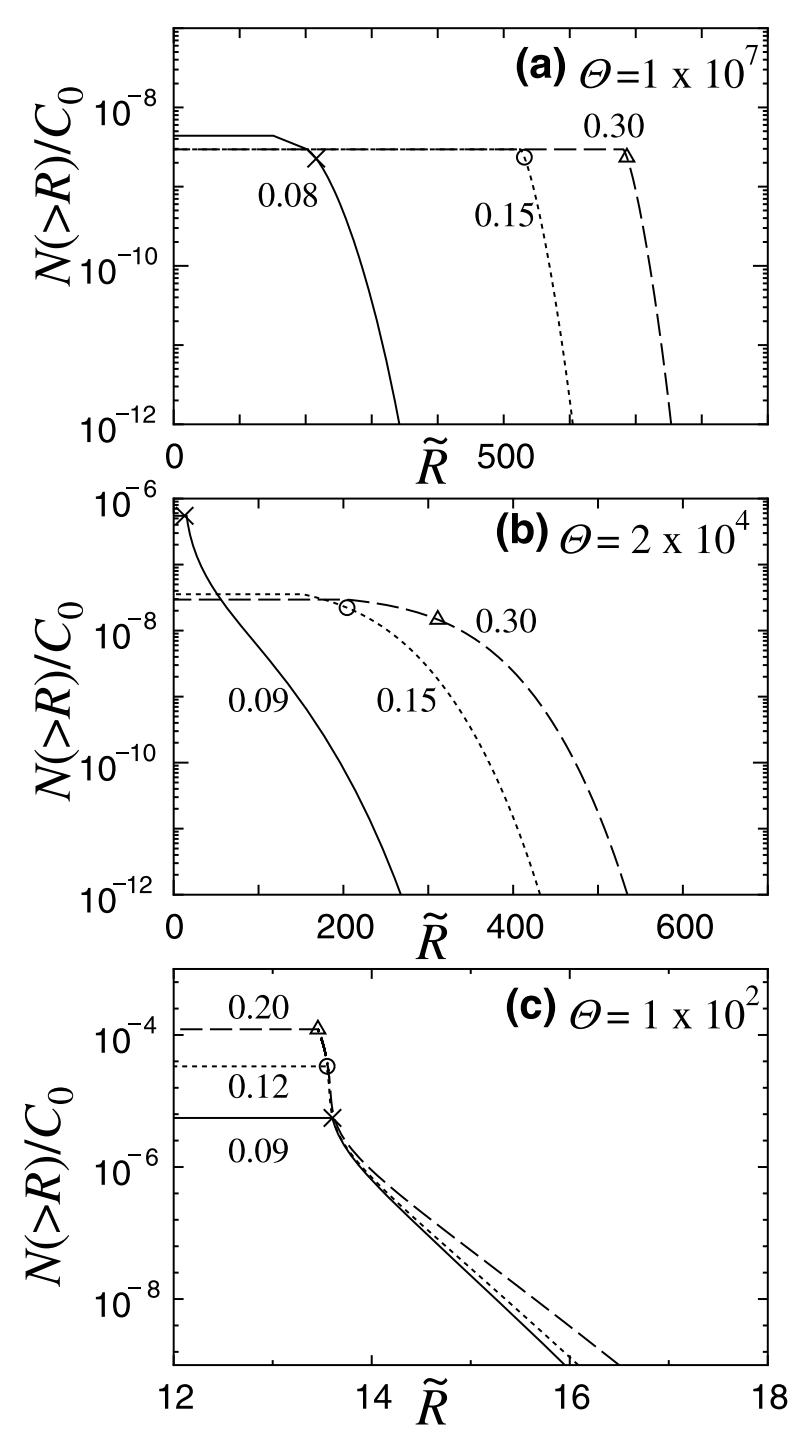

Fig. 14. Cumulative number densities of bubbles for $\Theta=1 \times 10^{7}$ (a), $2 \times 10^{4}(\mathrm{~b})$, and $1 \times 10^{2}(\mathrm{c})$, respectively. Panels (a), (b), and (c) correspond to the low, medium, and high viscosity systems, respectively. Other parameters than $\Theta$ are equal to reference values. In Panel (a), the solid, dotted, and dashed lines represent the cumulative number densities of bubbles at $x=0.08,0.15$, and 0.30, respectively. In Panel (b), the solid, dotted, and dashed lines represent the cumulative number densities of bubbles at $x=0.09,0.15$, and 0.30 , respectively. In Panel (c), the solid, dotted, and dashed lines represent the cumulative number densities of bubbles at $x=0.09,0.12$, and 0.20 , respectively. The cross, circule, and triangle on each curve indicate the size of bubbles at which the size distribution of bubbles in the normal style, $F(R)$, attains the maximum value.

dispersion at the same time. Noted that one can confirm in Fig. 14(b) that the cumulative number density in the small range of bubble size $(\tilde{R} \leq 50)$ decreases with time owing to the Ostwald ripening.

Bubble formation significantly depends on the size distribution of bubbles through the time-evolution of the volatile concentration. In the case that the size distribution of bubbles is an unimodal distribution with a small dispersion, bubbles with the average size have most of total volume of the gas phase. This case can be reproduced by Toramaru's model (1995). However, the size distribution like
Fig. 14(b) cannot be regarded as an unimodal distribution. Hence this causes the difference in the time-evolution of the volatile concentration between our model and Toramaru's model (1995) at the early stage.

The time-evolution of the cumulative number density of bubbles in the case of the extreme high viscosity is shown in Fig. 14(c). The solid, dotted, and dashed lines correspond to the cumulative number density of bubbles at $x=0.09$, 0.12 , and 0.20 , respectively. In the case of the extreme high viscosity, the cumulative number density of bubbles shows a rapidly decreasing function against the bubble size. Since liquid with the high viscosity hinders the bubble growth, a large number of bubbles are formed within the small size range.

\section{Discussion and Conclusions}

We investigated bubble formation in a viscous compressible liquid under decompression. Our results are useful in deriving informations from data of bubble vesiculation in experiments. This study is summarized as follows.

1) The effect of the Ostwald ripening on the bubble number density was explained clearly by taking into account the size distribution of bubbles. This study obviously pointed out the possibility that the number density of bubbles decreases in a different way from bubble coalescence. In region II, the Ostwald ripening leads to the considerable decrease in the number density of bubbles after bubble nucleation ceases. On the other hand, in region I, since the liquid viscosity prevents bubbles from growing, the number density of bubbles continues to increase. The volatile element in liquid is consumed only by bubbling. The Ostwald ripening does not have a serious effect on the timeevolution of the bubble number density in region III because bubbles can rapidly grow to the large size.

2) We derived analytical solutions to the threshold between regions II and III, $\Theta_{\text {II/III }}$, by using the analytical method of Yamada et al. (2005). The transition from regions III to II occurs when the expansion time of bubbles extends more than 0.6 times duration time of nucleation. Then, the threshold parameter $\Theta_{\mathrm{II} / \mathrm{III}}$, which is given by Eq. (49), is weakly sensitive to the diffusion coefficient and strongly to the surface tension. It is approximately proportional to $\tilde{\gamma}^{-3}$. In addition, analytical expression of the threshold between regions $\mathrm{I}$ and II, $\Theta_{\mathrm{I} / \mathrm{II}}$, was derived and is given by Eq. (50). In region I, the typical growth time of bubbles is large compared with the decompression time. The threshold parameter, $\Theta_{\mathrm{I} / \mathrm{II}}$, depends on weakly the diffusion coefficient and directly the surface tension. It is approximately proportional to $\tilde{\gamma}^{-1.5}$.

3) The time-evolutions of the bubble size distribution were examined. In the case of the low viscosity, the bubble size distribution evolves as an unimodal size distribution with the small dispersion. In the case of region II, the bubble size distribution has a different form from the case of the low viscosity just after the nucleation of bubbles ceases. This originates from the fact that the type of the bubble growth is exponential, as 

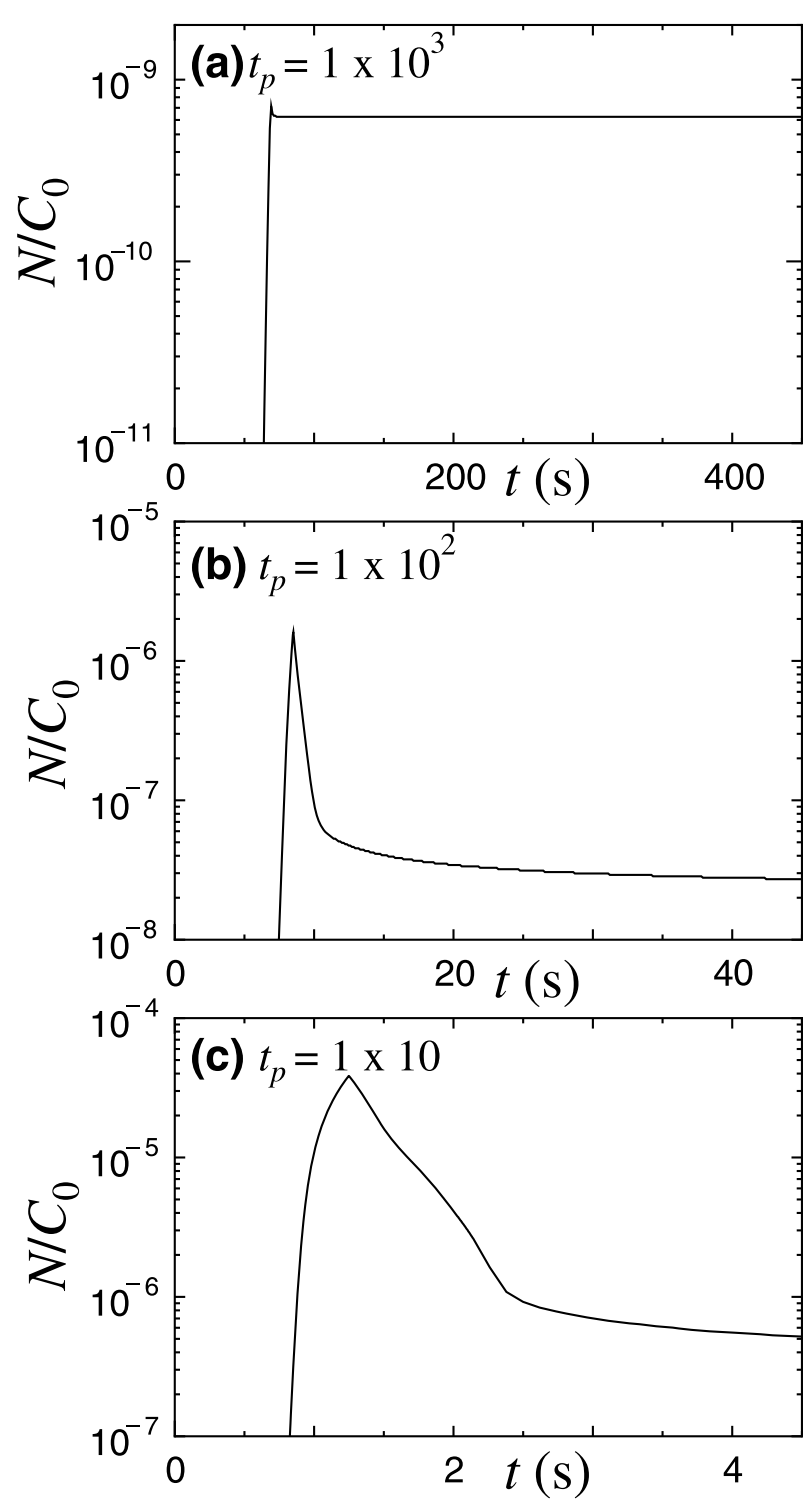

Fig. 15. Time-evolutions of the bubble number density for $t_{P}=1 \times 10^{3} \mathrm{~s}$ (a), $1 \times 10^{2} \mathrm{~s}(\mathrm{~b})$, and $1 \times 10 \mathrm{~s}(\mathrm{c})$, respectively. Note that the abscissa axis in this figure is not normalized. The viscosity is $5 \times 10^{5} \mathrm{~Pa} \cdot \mathrm{s}$ and the diffusion coefficient is $1 \times 10^{-13} \mathrm{~m}^{2} / \mathrm{s}$. Other parameters are equal to the reference values. In the case of the high decompression rate, the Ostwald ripening occurs and causes the larger decrease in the bubble number density.

indicated by Eq. (38). Even at the later stage, the size distribution has a wide dispersion. In the case of the extreme high viscosity, the bubble growth is prohibited by the viscosity and the size distribution of bubbles also becomes unimodal. Then, the radius of bubbles is as small as the critical radius and only the number density of bubbles increases with time. From these results, the size distribution of bubbles would become an unimodal size distribution when the void ratio approximately attains to 0.3 , except the middle range of the liquid viscosity. Under an unimodal size distribution, the relative velocity between bubbles is small and the bubble collision would scarcely occur in a hydrostatic state. Thus, the coalescence of bubbles is likely to take place owing to the breakup of liquid film when bubbles grow enough.

Since we focused the dependency of the diffusion coefficient and the viscosity on bubble formation, we regarded the variation of $\Theta$ and $\Lambda$ as that of the viscosity and the diffusion coefficient. However, noted that this variation of nondimensional parameters can be reread as the variation of the decompression rate which is a key parameter. In experiments, the decompression rate is one of freely controlled parameters. Hence, it is useful to show explicitly the numerical results of bubble formation for various decompression rates. The time-evolution of the bubble number density is shown in Fig. 15(a), (b), and (c) for $t_{P}=1 \times 10^{3} \mathrm{~s}, 1 \times 10^{2}$ $\mathrm{s}$, and $1 \times 10 \mathrm{~s}$, respectively. The viscosity and the diffusion coefficient are fixed as constant. As seen in Fig. 15, the bubble number density decreases after the number density of bubbles attains maximum. When the decompression rate is small (or $t_{P}$ is large), a number of nucleated bubbles is small and bubbles have the growth time enough before a super-saturated state is released. Therefore, the Ostwald ripening rarely occurs. The decompression rate plays a same role as the viscosity in the time-evolution of bubble nucleation.

In this study, we neglect the coalescence between bubbles. However, it is pointed out from the decompression experiments that the coalescence between bubbles leads to the decrease in the bubble number density (Larsen and Gardner, 2000; Larsen et al., 2004). In addition, the interaction between bubbles such as bubble coalescence significantly influences the time-evolutions of the size distribution (Gaonac'h et al., 1996). Thus, it is expected that bubble coalescence will change the size distribution of bubbles and the bubble number density at the later time. This problem is still remained. It is needed to quantitatively investigate the behavior of bubble coalescence in the future.

Acknowledgments. We wish to thank H. Tanaka and A. Toramaru for useful discussions and helpful comments. We also have benefited from comments by S. Takeuchi and S. Okumura. We thank A. Proussevitch and an anonymous reviewer for their insight comments that greatly improved the manuscript.

\section{Appendix A. Derivation of the Analytical Expres- sion of the Maximum Number Den- sity of Bubbles in Region I}

In the case of bubble nucleation in highly viscous liquid, the nucleation rate increases slowly after bubbles are formed to some extent. This means that the critical radius hardly varies. First we introduce the time $x_{1}$ when the increase speed of the nucleation rate begins to slow down.

The problem addressed here is to determine the time $x_{1}$ from which most bubbles begin to be formed in liquid with the extreme high viscosity. As found from Fig. 5, the behavior of the nucleation rate in the case of the high viscosity is divided into two phases, that is, the rapid increase phase and the slow increase phase of the nucleation rate. In order to derive an approximate expression, we assume that the nucleation rate in the rapid increase phase is given by $\tilde{J}_{0}(x)$, which is given by Eq. (36), and by a constant value in the slow increase phase, as indicated in Fig. A.1. In order to 


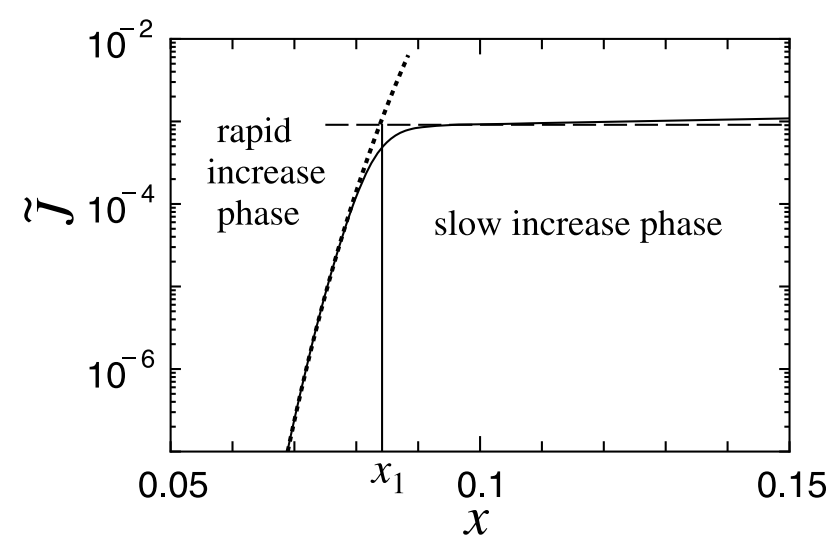

Fig. A.1. Time-evolution of the nucleation rate in the case of $\Theta=1 \times 10^{2}$. The other parameters are equal to reference values. The solid line represents the numerical result which is same as that of Fig. 5(b). As found from this figure, there are two phases that the nucleation rate increases rapidly and slowly. The rapid increase phase and the slow increase phase correspond to the period before and after the time $x_{1}$, respectively. In the rapid increase phase, the numerical result of the nucleation rate is approximately produced by $\tilde{J}_{0}(x)$ (dotted line), which is given by Eq. (36). On the other hand, it is represented as the constant value $\tilde{J}_{0}\left(x_{1}\right)$ (dashed line) in the slow increase phase. Since the slow increase phase is much longer than the rapid increase phase, many bubbles are formed in this phase. The time $x_{1}$ is given by Eq. (A.4) and displayed by the vertical solid line.

derive the expression of $x_{1}$, we make use of the analytical method of Yamada et al. (2005).

In highly viscous liquid, the bubble growth does not proceed much. So, it is reasonable that the radius of bubbles is assumed to be equal to the critical radius. Then, Eq. (23) is approximately expressed by

$$
\frac{\mathrm{d} \tilde{C}}{\mathrm{~d} x}=-\frac{\alpha \Lambda}{4 m} \sqrt{\frac{\tilde{\gamma}}{\pi^{3}}} \tilde{C}^{2} \tilde{R}_{\mathrm{cr}}^{2} \exp \left(-\frac{\tilde{\gamma}}{3} \tilde{R}_{\mathrm{cr}}^{2}\right)
$$

At the beginning of nucleation, the decrease in the volatile concentration is small. Therefore, we can assume that $|1-\tilde{C}| \ll 1$. Using this assumption, we obtain from Eq. (A.1)

$$
\begin{aligned}
\frac{\mathrm{d} \tilde{C}}{\mathrm{~d} x}= & -\frac{\alpha \Lambda \tilde{\gamma}^{2}}{9 m} \sqrt{\frac{\tilde{\gamma}}{\pi^{3}}}\left(e^{-\beta x}-1+x\right)^{-2} \\
& \cdot \exp \left[-\frac{4 \tilde{\gamma}^{3}}{27}\left(e^{-\beta x}-1+x\right)^{-2}\right] .
\end{aligned}
$$

In this analytical method, the assumption that $x \ll 1$ is needless.

In region I, the significant feature of the time-evolution of nucleation is that there are the rapid $\left(x<x_{1}\right)$ and slow increase phases $\left(x>x_{1}\right)$. This means that the time variation of the supersaturation rate, $(\mathrm{d} S / \mathrm{d} x)_{x \geq x_{1}}$, is small compared with $(\mathrm{d} S / \mathrm{d} x)_{x<x_{1}}$. So, we assume for simplicity that in the slow increase phase

$$
\left(\frac{\mathrm{d} S}{\mathrm{~d} x}\right)_{x \geq x_{1}}=0
$$

Substituting Eq. (3) into Eq. (A.3) and using Eq. (A.2), the approximate expression of $x_{1}$ is provided by

$$
\begin{aligned}
& \frac{\alpha \Lambda \tilde{\gamma}^{2}}{9} \sqrt{\frac{\tilde{\gamma}}{\pi^{3}}}\left(e^{-\beta x_{1}}-1+x_{1}\right)^{-2} \\
& \cdot \exp \left[-\frac{4 \tilde{\gamma}^{3}}{27}\left(e^{-\beta x_{1}}-1+x_{1}\right)^{-2}\right] \\
& +\beta-\frac{1}{1-x_{1}}=0 .
\end{aligned}
$$

As found in Eq. (A.4), the time $x_{1}$ depends on $\Lambda$. The time $x_{1}$ solved from Eq. (A.4) is 0.084 for the parameter set of Fig. 5.

Bubbles nucleated at $x>x_{1}$ have a great effect on the number density of bubbles and the void ratio. Therefore, the number density of bubbles is approximately given as the analytical form in region I by

$$
\frac{N(x)}{C_{0}} \simeq \tilde{J}_{0}\left(x_{1}\right)\left(x-x_{1}\right) .
$$

On the other hand, the void ratio $\phi$ is defined by

$$
\phi=\alpha \int_{0}^{x} \tilde{R}^{3}\left(x, x^{\prime}\right) \tilde{J}\left(x^{\prime}\right) \mathrm{d} x^{\prime} .
$$

Assuming that the bubble radius is equal to the critical radius at $x=x_{1}, \tilde{R}_{\mathrm{cr}}\left(x_{1}\right)$, Eq. (A.6) is given by

$$
\phi \simeq \alpha \tilde{R}_{\mathrm{cr}}^{3}\left(x_{1}\right) \tilde{J}_{0}\left(x_{1}\right)\left(x-x_{1}\right),
$$

where the critical radius is approximately given under only the assumption that $|1-\tilde{C}| \ll 1$ by

$$
\tilde{R}_{\mathrm{cr}}\left(x_{1}\right)=\frac{2 \tilde{\gamma}}{3}\left(e^{-\beta x_{1}}-1+x_{1}\right)^{-1} .
$$

Substituting Eq. (A.7) into Eq. (A.5), we obtain as another form

$$
\frac{N^{\mathrm{I}}(x)}{C_{0}} \simeq \frac{\phi}{\alpha \tilde{R}_{\mathrm{cr}}^{3}\left(x_{1}\right)} .
$$

The analytical maximum number densities of bubbles are about $1.2 \times 10^{-3}$ and $6.9 \times 10^{-3}$ in the cases of $\tilde{\gamma}=0.88$ and 2.64, respectively, for $\phi=0.3$ and $\Lambda=9 \times 10^{7}$. It is noted that Eq. (A.9) is weak sensitive to $\Lambda$ through $x_{1}$.

\section{Appendix B. Derivation of Eq. (38)}

In liquid with the large viscosity, the driving force of bubble growth is mainly the bubble expansion. The large viscosity suppresses the bubble growth and as a result the internal pressure remains high before the bubble expansion. When the difference between the internal and external pressure is large to some extents, bubbles expand.

The internal pressure is given from Eqs. (1) and (2) as

$$
\frac{\tilde{P}_{\mathrm{gas}}}{\tilde{P}}=\left(\frac{\tilde{C}_{\mathrm{in}}}{\tilde{C}_{\mathrm{eq}}}\right)^{m}
$$

Now, we assume that $\tilde{C}_{\text {in }}$ is set to be unity. This assumption means that the internal pressure is very high. Using Eq. (35), the approximative expression of $\tilde{P}_{\text {gas }}$ is given by

$$
\tilde{P}_{\text {gas }}=1-\beta x
$$


where the higher order terms of $x$ than second are neglected. Substituting Eq. (B.2) into Eq. (22), we have

$$
\frac{\mathrm{d} \tilde{R}\left(x, x^{\prime}\right)}{\mathrm{d} x}=\frac{\Theta}{4} \tilde{R}\left[(1-\beta) x-\frac{2 \tilde{\gamma}}{3 \tilde{R}}\right] .
$$

Deriving the general and particular solutions of the differential equation (B.3) by use of method of variation of parameters, we obtain Eq. (38). It is noted that the general solution of equation (B.3) was provided by Lensky et al. (2004). Then, we use the initial condition that $\tilde{R}\left(x^{\prime}, x^{\prime}\right)=\tilde{R}_{\mathrm{cr}}$. Under the zero-th order approximation $(|1-\tilde{C}| \ll 1$ and $x \ll 1)$, the critical radius is given by

$$
\tilde{R}_{\mathrm{cr}}(x)=\frac{2 \tilde{\gamma}}{3(1-\beta) x} .
$$

In addition, in Eq. (39), erf( $x$ ) is the error function defined by

$$
\operatorname{erf}(x)=\frac{2}{\sqrt{\pi}} \int_{0}^{x} e^{-y^{2}} \mathrm{~d} y .
$$

\section{Appendix C. Derivation of Eq. (43)}

The derivation of Eq. (43) can be solved straightforward. The non-dimensional time $x_{J}$ is given from the condition that

$$
\left(\frac{\mathrm{d} S}{\mathrm{~d} x}\right)_{x=x_{J}}=0
$$

The supersaturation ratio is given under the zero-th order approximation by

$$
S(x)=\tilde{C}(x)\left\{1+\frac{(1-\beta)}{m} x\right\},
$$

where Eq. (35) is used. Differentiating Eq. (C.2) and using Eq. (C.1), we have

$$
\frac{1}{\tilde{C}}\left(\frac{\mathrm{d} \tilde{C}}{\mathrm{~d} x}\right)_{x=x_{J}}=-\frac{1-\beta}{m}\left(1+\frac{1-\beta}{m} x\right)^{-1} .
$$

Now, we assume that $x \ll 1$ and $|1-\tilde{C}| \ll 1$. These assumptions enable us to reduce Eq. (C.3) to a simpler form:

$$
\left(\frac{\mathrm{d} \tilde{C}}{\mathrm{~d} x}\right)_{x=x_{J}}=-\frac{1-\beta}{m}
$$

Substituting the differential form of Eq. (42) into Eq. (C.4), we obtain Eq. (43). Then, it is assumed that $\tilde{R}^{\prime}\left(x, x_{0}\right)$ varies slow compared with the exponential function. Moreover, we also assume that $2 b^{2} \gg 1 / x$.

\section{Appendix D. Derivation of Eq. (49)}

The threshold from regions II to III is defined from the behavior of the maximum number density of bubbles. The threshold corresponds to the viscosity at which Eq. (46) is equivalent to Eq. (47), that is, $N_{\max }^{\mathrm{II}}=N_{\max }^{\mathrm{III}}$. Equation (46) represents the maximum number density of bubbles in region II while Eq. (47) reproduces the maximum number density of bubbles in region III. Equating Eq. (47) to Eq. (46), we obtain

$$
\left(\frac{x_{J}}{x_{0}}\right)^{2} \exp \left[\left(\frac{x_{0}}{x_{J}}\right)^{4}-1\right]=1
$$

where the values of $x_{J}$ in both Eqs. (46) and (47) are assumed to be equivalent. Solving this transcendental equation, we have

$$
\frac{x_{0}}{x_{J}} \simeq 0.864
$$

After some transformations, Eq. (49) is finally obtained. In Eq. (49), the value of $x_{J}$ is evaluated from Eq. (48).

\section{References}

Adams, G. W., J. L. Schmitt, and R. A. Zalabsky, The homogeneous nucleation of nonane, J. Chem. Phys., 81, 5074-5078, 1984.

Anderson, R. J., R. C. Miller, J. L. Kassner Jr., and D. E. Hagen, A study of homogeneous condensation-freezing nucleation of small water droplets in an expansion cloud chamber, J. Atmos. Sci., 37, 2508-2520, 1980.

Avrami, M., Kinetics of Phase Change I: General Theory, J. Chem. Phys., 7, 1103-1112, 1939.

Avrami, M., Kinetics of Phase Change II: Transformation-Time Relations for Random Distribution of Nuclei, J. Chem. Phys., 8, 212-224, 1940.

Avrami, M., Granulation, Phase Change, and Microstructure: Kinetics of Phase Change III, J. Chem. Phys., 9, 177-184, 1941.

Bagdassarov, N., A. Dorfman, and D. B. Dingwell, Effect of alkalis, phosphorus, and water on the surface tension of haplogranite melt, Am. Mineral., 85, 33-40, 2000.

Batchelor, G. K., An introduction to fluid dynamics, Cambridge University Press, 1967.

Blower, J. D., Factors controlling permeability-porosity relationships in magma, Bull. Volcanol., 63, 497-504, 2001.

Burnham, C. W., Water and magmas: A mixing model, Geochim. Cosmochim. Acta., 39, 1077-1084, 1975.

Dillmann, A. and G. E. A. Meier, A refined droplet approach to the problem of homogeneous nucleation from the vapor phase, J. Chem. Phys., 94, 3872-3884, 1991.

Epel'baum, M. B., I. V. Babashov, and T. P. Salova, Surface tension of felsic magmatic melts at high temperatures and pressure, Geochem. Int., 10, 343-345, 1973.

Feder, J., K. C. Russell, J. Lothe, and G. M. Pound, Homogeneous nucleation and growth of droplets in vapours, Adv. Phys., 15, 111-178, 1966.

Fogel, R. A. and M. J. Rutherford, The solubility of carbon dioxide in rhyolitic melt: A quantitative FTIR study, Am. Mineral., 75, 1311-1326, 1990.

Gaonac'h, H., S. Lovejoy, J. Stix, and D. Schertzer, A scaling growth model for bubbles in basaltic lava flows, Earth Planet. Sci. Lett., 139, 395-409, 1996.

Gardner, J. E., Heterogeneous bubble nucleation in highly viscous silicate melts during instantaneous decompression from high pressure, Chem. Geol., 236, 1-12, 2007.

Gardner, J. E., M. Hilton, and M. R. Carroll, Experimental constraints on degassing of magma: isothermal bubble growth during continuous decompression from high pressure, Earth Planet. Sci. Lett., 168, 201218, 1999.

Gardner, J. E., M. Hilton, and M. R. Carroll, Bubble growth in highly viscous silicate melts during continuous decompression from high pressure, Geochim. Cosmochim. Acta, 64, 1473-1483, 2000.

Ghiorso, M. S., I. S. E. Carmichael, M. L. Rivers, and R. O. Sack, The Gibbs free energy of mixing of natural silicate liquid; an expanded regular solution approximation for the calculation of magmatic intensive variables, Contrib. Mineral. Petrol., 84, 107-145, 1983.

Hess, K.-U. and D. B. Dingwell, Viscosities of hydrous leucogranitic melts: a non-Arrhenian model, Am. Mineral., 81, 1297-1300, 1996.

Hurwitz, S. and O. Navon, Bubble nucleation in rhyolitic melts: experiments at high pressure, temperature, and water content, Earth Planet. Sci. Lett., 122, 267-280, 1994.

Johnson, W. A. and R. F. Mehl, Reaction kinetics in processes of nucleation and growth, Am. Inst. Min. Metal. Petro. Eng., 135, 416-458, 1939.

Khitarov, N. I., Ye. B. Lebedev, A. M. Dorfman, and N. Sh. Bagdassarov, Effects of temperature, pressure, and volatiles on the surface tension of molten basalt, Geochem. Int., 16, 78-86, 1979.

Kikuchi, R., Treatment of droplike clusters in nucleation theory using Reiss's method, J. Stat. Phys., 3, 331-345, 1971. 
Klug, C. and K. V. Cashman, Vesiculation of May 18, 1980 Mount St. Helens magma, Geology, 22, 468-472, 1994.

Landau, L. D. and E. M. Lifshitz, Fluid Mechanics, 2nd edition, Butterworth-Heinemann, Boston, 1987.

Larsen, J. F. and J. E. Gardner, Experimental constraints on bubble interactions in rhyolite melt: implications for vesicle size distributions, Earth Planet. Sci. Lett., 180, 201-214, 2000.

Larsen, J. F., M.-H. Denis, and J. E. Gardner, Experimental study of bubble coalescence in rhyolitic and phonolitic melts, Geochim. Cosmochim. Acta, 68(2), 333-344, 2004.

Lensky, N. G., V. Lyakhovsky, and O. Navon, Radial variations of melt viscosity around growing bubbles and gas overpressure in vesiculating magmas, Earth Planet. Sci. Lett., 186, 1-6, 2001.

Lensky, N. G., O. Navon, and V. Lyakhovsky, Bubble growth during decompression of magma: experimental and theoretical investigation, $J$. Volcanol. Geotherm. Res., 129(1-3), 7-22, 2004.

Lifshitz, E. M. and L. P. Pitaevskii, Physical Kinetics, Pergamon, New York, 1981.

Lothe, J. and G. M. Pound, Reconsiderations of nucleation theory, J. Chem. Phys., 36, 2080-2085, 1962.

Lyakhovsky, V., S. Hurwitz, and O. Navon, Bubble growth in rhyolitic melts: Experimental and numerical investigation, Bull. Volcanol., 58, 19-32, 1996

Mangan, M. T. and T. Sisson, Delayed, disequilibrium degassing in rhyolite magma: decompression experiments and implications for explosive volcanism, Earth Planet. Sci. Lett., 183, 441-455, 2000.

Mangan, M. T., L. Mastin, and T. Sisson, Gas evolution in eruptive conduits: combining insights from high temperature and pressure decompression experiments with steady-state flow modeling, J. Volcanol. Geotherm. Res., 129, 23-36, 2004a.

Mangan, M. T., T. W. Sisson, and W. B. Hankins, Decompression experiments identify kinetic controls on explosive silicic eruptions, Geophys. Res. Lett., 31, L08605, 2004b.

Mourtada-Bonnefoi, C. C. and D. Laporte, Experimental study of homogeneous bubble nucleation in rhyolitic magmas, Geophys. Res. Lett., 26, 3505-3508, 1999.

Mourtada-Bonnefoi, C. C. and D. Laporte, Homogeneous bubble nucleation in rhyolitic magmas: an experimental study of the effect of $\mathrm{H}_{2} \mathrm{O}$ and $\mathrm{CO}_{2}$, J. Geophys. Res., 107(B4), ECV 2-1-2-21, 2002.

Mourtada-Bonnefoi, C. C. and D. Laporte, Kinetics of bubble nucleation in a rhyolitic melt: an experimental study of the effect of ascent rate, Earth Planet. Sci. Lett., 218, 521-537, 2004.

Noguchi, S., A. Toramaru, and T. Shimano, Crystallization of microlites and degassing during magma ascent: Constraints on the fluid mechanical behavior of magma during the Tenjo Eruption on Kozu Island, Japan, Bull. Volcanol., 68(5), 432-449, 2006.

Nowak, M. and H. Behrens, An experimental investigation on diffusion of water in haplogranitic melts, Contrib. Mineral. Petrol., 126, 4, 365-376, 1997.

Papale, P., Modeling of the solubility of a two-component $\mathrm{H}_{2} \mathrm{O}+\mathrm{CO}_{2}$ fluid in silicate liquids, Am. Mineral., 84, 477-492, 1999.

Papale, P., Volcanic conduit dynamics, FROM MAGMA TO TEPHRA, Modelling Physical Processes of Explosive Volcanic Eruptions, Elsevier, chapter 3, 2001.

Pradell, T., D. Crespo, N. Clavaguera, and M. T. Clavaguera-Mora, Diffusion controlled grain growth in primary crystallization: Avrami expo- nents revised, J. Phys., Condens. Matter, 10, 3833-3844, 1998.

Proussevitch, A. A. and D. L. Sahagian, Dynamics and energetics of bubble growth in magmas: analytical formulation and numerical modeling, J. Geophys. Res., 103(B8), 18,223-18,251, 1998.

Proussevitch, A. A., D. L. Sahagian, and A. T. Anderson, Dynamics of diffusive bubble growth in magmas: isothermal case, J. Geophys. Res., 98(B12), 22,283-22,307, 1993.

Proussevitch, A. A., D. L. Sahagian, and E. P. Tsentalovich, Statistical analysis of bubble and crystal size distributions: Formulations and procedures, J. Volcanol. Geotherm. Res., 164(3), 95-111, 2007a.

Proussevitch, A. A., D. L. Sahagian, and W. D. Carlson, Statistical analysis of bubble and crystal size distributions: Application to Colorado Plateau basalts, J. Volcanol. Geotherm. Res., 164(3), 112-126, $2007 \mathrm{~b}$.

Shimano, T. and S. Nakada, Vesiculation path of ascending magma in the 1983 and the 2000 eruptions of Miyakejima volcano, Japan, Bull. Volcanol., 68(6), 549-566, 2006.

Sparks, R. S. J., The dynamics of bubble formation and growth in magmas: a review and analysis, J. Volcanol. Geotherm. Res., 3, 1-37, 1978.

Takeuchi, S., S. Nakashima, A. Tomiya, and H. Shinohara, Experimental constraints on the low gas permeability of vesicular magma during decompression, Geophys. Res. Lett., 32, L10312, 2005.

Tanaka, K. K., H. Tanaka, and K. Nakazawa, Non-equilibrium condensation in a primordial solar nebula: formation of refractory metal nuggets, Icarus, 160, 197-207, 2002.

Toramaru, A., Vesiculation process and bubble size distributions in ascending magmas with constant velocities, J. Geophys. Res., 94(B12), 17,523-17,542, 1989.

Toramaru, A., Numerical study of nucleation and growth of bubbles in viscous magmas, J. Geophys. Res., 100, 1913-1931, 1995.

Toramaru, A., BND (bubble number density) decompression rate meter for explosive volcanic eruptions, J. Volcanol. Geotherm. Res., 154, 303316, 2006.

Yamada, K., Size distribution of bubbles nucleated in viscous liquid, Tokyo institute of Technology, Ph.D. thesis, 2005.

Yamada, K., H. Tanaka, K. Nakazawa, and H. Emori, A new theory bubble formation in magma, J. Geophys. Res., 110, B02203, 2005.

Yamada, K., H. Emori, and K. Nakazawa, Bubble expansion rates in viscous compressible liquid, Earth Planets Space, 58, 865-872, 2006.

Yamamoto, T. and H. Hasegawa, Grain formation through nucleation process in astrophysical environment, Prog. Theo. Phys., 58(3), 816-828, 1977.

Yamamoto, T., T. Chigai, S. Watanabe, and T. Kozasa, Kinetic theory of steady chemical nucleation in the gas phase, Astron. Astrophys., 380, 373-383, 2001.

Zhang, $\mathrm{Y} ., \mathrm{H}_{2} \mathrm{O}$ in rhyolitic glasses and melts: measurement, speciation, solubility, and diffusion, Rev. Geophys., 37, 493-516, 1999.

Zhang, Y. and $\mathrm{H}$. Behrens, $\mathrm{H}_{2} \mathrm{O}$ diffusion in rhyolitic melts and glasses, Chem. Geol., 169, 243-262, 2000.

Zhang, Y., X. Zhengjiu, Z. Mengfan, and W. Haoyue, Silicate melt properties and volcanic eruptions, Rev. Geophys., 45(4), DOI:10.1029/ 2006RG000216, 2007.

K. Yamada (e-mail: k-yamada@aoni.waseda.jp), H. Emori, and K. Nakazawa 